\title{
Double Lie algebroids and representations up to homotopy
}

\author{
A. Gracia-Saz ${ }^{1}$ (D) M. Jotz Lean ${ }^{2,3}$ (ID $\cdot$ \\ K. C. H. Mackenzie ${ }^{2}(D)$ R. A. Mehta ${ }^{4}$
}

Received: 26 January 2016 / Accepted: 25 May 2017 / Published online: 7 July 2017

C The Author(s) 2017. This article is an open access publication

\begin{abstract}
We show that a double Lie algebroid, together with a chosen decomposition, is equivalent to a pair of 2-term representations up to homotopy satisfying compatibility conditions which extend the notion of matched pair of Lie algebroids. We discuss in detail the double Lie algebroids arising from the tangent bundle of a Lie algebroid and the cotangent bundle of a Lie bialgebroid.
\end{abstract}

Keywords Double Lie algebroids · Representations up to homotopy · Matched pairs

Mathematics Subject Classification Primary 53D17; Secondary 17B66 - 18D05

Communicated by Jim Stasheff.

K. C. H. Mackenzie

K.Mackenzie@sheffield.ac.uk

A. Gracia-Saz

alfonso@math.toronto.edu

M. Jotz Lean

M.Jotz-Lean@sheffield.ac.uk; madeleine.jotz-lean@mathematik.uni-goettingen.de

R. A. Mehta

rmehta@smith.edu

1 Department of Mathematics, University of Toronto, Toronto, ON M5S 2E4, Canada

2 School of Mathematics and Statistics, The University of Sheffield, Sheffield S3 7RH, UK

3 Present Address: Mathematisches Institut, Georg-August Universität Göttingen, Bunsenstraße 3-5, 37073 Göttingen, Germany

4 Department of Mathematics and Statistics, Smith College, 44 College Lane, Northampton, MA 01063, USA 


\section{Contents}

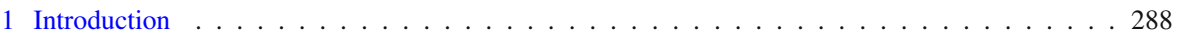

2 Background and definitions . . . . . . . . . . . . . . . . . . . . . 290

2.1 Double vector bundles, decompositions and dualization . . . . . . . . . . . . . . . . 290

2.2 LA-vector bundles and double Lie algebroids . . . . . . . . . . . . . . . . . . . . . . 295

2.3 Representations up to homotopy and LA-vector bundles . . . . . . . . . . . . . . . . 296

3 Main theorem and examples . . . . . . . . . . . . . . . . . . . . . . . . . . . . . . 299

3.1 Matched pairs of 2-representations and main result f . . . . . . . . . . . . . 300

3.2 Comparison with the equations of Voronov . . . . . . . . . . . . . . . . . 301

3.3 The tangent double vector bundle of a Lie algebroid . . . . . . . . . . . . . . . . . 304

3.4 The cotangent double of a Lie bialgebroid . . . . . . . . . . . . . . . . . 307

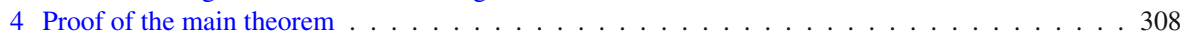

4.1 Families of sections of Lie bialgebroids . . . . . . . . . . . . . . . . . . . . . . . 309

4.2 The Lie bialgebroid conditions on lifts and on core sections . . . . . . . . . . . . . . 311

References . . . . . . . . . . . . . . . . . . . . . . . . . 318

\section{Introduction}

Double Lie algebroids first arose as the infinitesimal form of double Lie groupoids [11, 14]. In the same way as the Lie theory of Lie groupoids and Lie algebroids expresses many of the basic infinitesimalization and integration results of differential geometry, the process of taking the double Lie algebroid of a double Lie groupoid captures two-stage differentiation processes, such as the iterated tangent bundle of a smooth manifold, and the relations between a Poisson Lie group, its Lie bialgebra and its symplectic double groupoid.

The transition from a double Lie groupoid to its double Lie algebroid is straightforward. To define an abstract concept of double Lie algebroid, however, is much more difficult, since there is no meaningful way in which a Lie algebroid bracket can be said to be a morphism with respect to another Lie algebroid structure. The solution ultimately found was to extend the duality between Lie algebroids and Lie-Poisson structures to the double context, using the duality properties of double vector bundles [16]. This definition was given a simple and elegant reformulation in terms of super geometry by Th. Voronov [26]. In terms of super geometry, an ordinary Lie algebroid structure on a vector bundle corresponds to a homological vector field $Q$ of weight 1 on the parity-reversed bundle. A double vector bundle $D$ with Lie algebroid structures on both bundle structures on $D$ therefore involves two homological vector fields and if these are of suitable weights with respect to the grading from the double vector bundle structure, the main compatibility condition of [26] is that they commute.

In the present paper we give a third formulation of double Lie algebroids, in terms of representations up to homotopy as defined in [1,4]; this differs from the concept of the same name introduced in [3].

In fact the representations up to homotopy which are relevant are concentrated in degrees 0 and 1 and we refer to them as 2-representations for brevity (see Definition 2.8). A 2-representation is a special form of Quillen's notion of superconnection; see below following 2.7. We could paraphrase the content of the paper as showing 
that double Lie algebroids can be regarded as matched pairs of flat Lie algebroid superconnections.

We now give a more detailed description of the background to the paper. Consider first a double vector bundle $D$ with Lie algebroid structures on two parallel sides, which are compatible with the vector bundle structures on the other sides; these are the LA-vector bundles of [16, Definition 3.3] and, in an equivalent reformulation, the VB-algebroids of [4]. Further suppose that $D$ is 'decomposed'; that is, as a manifold it is the fibre product $A \times_{M} B \times_{M} C$ of three vector bundles $A, B, C$ on the same base $M$, and the vector bundle structures on $D$ are the pullbacks of $B \oplus C$ to $A$ and of $A \oplus C$ to $B$. Then [4] showed that LA-vector bundle structures on $D$ are in bijective correspondence with 2-representations defined in terms of $A, B$ and $C$.

Decompositions of double vector bundles may themselves be regarded as extensions of the notion of connection. One definition of a connection in a vector bundle $(E, q, M)$ is a map C: $E \times_{M} T M \rightarrow T E$ which is linear both as a map from the pullback of $T M$ over the projction $E \rightarrow M$ and as a map from the pullback of $E$ over the projection $T M \rightarrow M$, and which is right-inverse to both projections. This formulation, which can be found in Dieudonné, is precisely a decomposition of $T E$ in our sense. However for a decomposition of a double vector bundle to possess connection-like properties it is necessary for there to be bracket structures on at least one pair of parallel sides; that is, for it to have a LA-vector bundle structure.

We assume, as part of the definition of a double vector bundle, that a decomposition exists; this property is preserved by the various prolongation and dualization processes studied here and in the references. Decompositions may be regarded as trivializations of $D$ at the double level; in this paper we do not need to trivialize $A, B$ and $C$. For a formulation in coordinate terms, see [26].

Now consider an arbitrary double Lie algebroid $D$. The Lie algebroid structures on $D$ may be considered as a pair of LA-vector bundle structures and accordingly a decomposition of $D$ expresses the double Lie algebroid structure as a pair of 2-representations. Our main result (Theorem 3.6) determines the compatibility conditions between these and, conversely, proves that a suitable pair of 2-representations defines a double Lie algebroid structure on $D$.

Our formulation is significantly different from both the original formulation and that of [26]. Our treatment resembles the coordinate treatment in [26] inasmuch as the three intrinsic conditions of [16] are replaced by a greater number of conditions which are dependent on auxiliary data, but which are easier to work with. On the other hand, our methods are entirely 'classical' rather than supergeometric, and rely on a global decomposition rather than a local trivialization.

Our formulation may also be regarded as a considerable generalization of the description of a vacant double Lie algebroid in terms of a matched pair of representations $[16, \S 6]$ - that is, of representations of Lie algebroids in the strict sense, without curvature. For this reason we regard the conditions (M1) to (M7) in Definition 3.1 as defining a matched pair of 2-representations.

In turn, [7] shows that the bicrossproduct of a matched pair of 2-representations is a split Lie 2-algebroid, in the same way that the bicrossproduct of a matched pair of representations of Lie algebroids is a Lie algebroid $[10,18]$. In a different direction, 
[8] will apply our main result to show that double Lie algebroids which are transitive in a sense appropriate to the double structure are determined by a simple diagram of morphisms of ordinary Lie algebroids.

We now describe the contents of the paper.

In Sect. 2 we recall the basic notions needed throughout the paper. We begin with double vector bundles, the special classes of sections with which it is easiest to work, and the nonstandard pairing between their duals. In Sect. 2.2 we recall LA-vector bundles and double Lie algebroids, and in Sect. 2.3 we finally define 2-representations.

In Sect. 3 we state our main result and apply it to the double Lie algebroids which arise from the tangent and cotangent prolongations of a Lie algebroid. The main work of the proof of Theorem 3.6 is given in Sect. 4 .

We have included definitions of the key concepts required; in particular it is not necessary to have a detailed knowledge of $[1,4,16]$ or $[26]$.

\section{Background and definitions}

\subsection{Double vector bundles, decompositions and dualization}

We briefly recall the definitions of double vector bundles, of their linear and core sections, and of their linear splittings and lifts. We refer to $[4,15,20]$ for more detailed treatments.

Definition 2.1 A double vector bundle is a commutative square

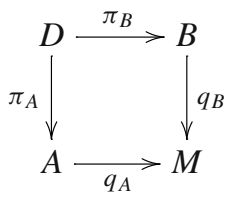

satisfying the following four conditions:

1. all four sides are vector bundles;

2. $\pi_{B}$ is a vector bundle morphism over $q_{A}$;

3. $+_{B}: D \times_{B} D \rightarrow D$ is a vector bundle morphism over $+: A \times_{M} A \rightarrow A$, where $+_{B}$ is the addition map for the vector bundle $D \rightarrow B$;

4. the scalar multiplication $\mathbb{R} \times D \rightarrow D$ in the bundle $D \rightarrow B$ is a vector bundle morphism over the scalar multiplication $\mathbb{R} \times A \rightarrow A$,

together with a fifth condition (5) below.

The corresponding statements for the operations in the bundle $D \rightarrow A$ follow.

Given a double vector bundle $(D ; A, B ; M)$, the vector bundles $A$ and $B$ are called the side bundles. The core $C$ of a double vector bundle is the intersection of the kernels of $\pi_{A}$ and $\pi_{B}$. It has a natural vector bundle structure over $M$, the restriction of either structure on $D$, the projection of which we call $q_{C}: C \rightarrow M$. The inclusion $C \hookrightarrow D$ is usually denoted by 


$$
C_{m} \ni c \longmapsto \bar{c} \in \pi_{A}^{-1}\left(0_{m}^{A}\right) \cap \pi_{B}^{-1}\left(0_{m}^{B}\right)
$$

Given any three vector bundles $A, B, C$ on the same base manifold $M$, the fibre product $\bar{D}:=A \times{ }_{M} B \times{ }_{M} C$ has a natural structure of double vector bundle with side bundles $A$ and $B$ and core $C$; the vector bundle structure on $\bar{D} \rightarrow A$ is the pullback of $B \oplus C$ to $A$ and likewise the vector bundle structure on $\bar{D} \rightarrow B$ is the pullback of $A \oplus C$ to $B$. We can now state the fifth condition of Definition 2.1:

5. there is a diffeomorphism from $D$ to the double vector bundle $\bar{D}=A \times{ }_{M} B \times{ }_{M} C$, where $C$ is the core of $D$, which is the identity on $A, B$ and $C$ and is an isomorphism of vector bundles $D \rightarrow \bar{D}$ over $A$ and $B$.

A morphism of double vector bundles from $(D ; A, B ; M)$ to $\left(D^{\prime} ; A^{\prime}, B^{\prime} ; M^{\prime}\right)$ consists of smooth maps $\phi: D \rightarrow D^{\prime}, \phi_{A}: A \rightarrow A^{\prime}, \phi_{B}: B \rightarrow B^{\prime}$ and $\phi_{M}: M \rightarrow M^{\prime}$ such that each of $\left(\phi, \phi_{A}\right),\left(\phi, \phi_{B}\right),\left(\phi_{A}, \phi_{M}\right)$ and $\left(\phi_{B}, \phi_{M}\right)$ is a vector bundle morphism.

Given a double vector bundle $(D ; A, B ; M)$, the space of sections $\Gamma_{B}(D)$ is generated as a $C^{\infty}(B)$-module by two distinguished classes of sections (see [16]), the linear and the core sections which we now describe.

Definition 2.2 For a section $c: M \rightarrow C$, the corresponding core section $c^{\dagger}: B \rightarrow D$ is defined as

$$
c^{\dagger}\left(b_{m}\right)=\tilde{0}_{b_{m}}+{ }_{A} \overline{c(m)}, \quad m \in M, b_{m} \in B_{m} .
$$

We denote the corresponding core section $A \rightarrow D$ by $c^{\dagger}$ also, relying on the argument to distinguish between them.

Definition 2.3 A section $\xi \in \Gamma_{B}(D)$ is called linear if $\xi: B \rightarrow D$ is a bundle morphism from $B \rightarrow M$ to $D \rightarrow A$ over a section $a \in \Gamma(A)$.

The space of core sections of $D$ over $B$ will be written $\Gamma_{B}^{c}(D)$ and the space of linear sections $\Gamma_{B}^{\ell}(D)$. Given $\psi \in \Gamma\left(B^{*} \otimes C\right)$, there is a linear section $\widetilde{\psi}: B \rightarrow D$ over the zero section $0^{A}: M \rightarrow A$ given by

$$
\widetilde{\psi}\left(b_{m}\right)=\widetilde{0}_{b_{m}}+{ }_{A} \overline{\psi\left(b_{m}\right)} .
$$

We call $\widetilde{\psi}$ a core-linear section.

Example 2.4 Let $A, B, C$ be vector bundles over $M$ and consider $\bar{D}=A \times{ }_{M} B \times{ }_{M} C$ with the vector bundle structures described in Definition 2.1. We call a double vector bundle of this type decomposed or a trivial double vector bundle with core $C$. The core sections are given by

$$
c^{\dagger}: b_{m} \mapsto\left(0_{m}^{A}, b_{m}, c(m)\right), \quad \text { where } m \in M, \quad b_{m} \in B_{m}, \quad c \in \Gamma(C),
$$

and similarly for $c^{\dagger}: A \rightarrow D$. The space of linear sections $\Gamma_{B}^{\ell}(D)$ is naturally identified with $\Gamma(A) \oplus \Gamma\left(B^{*} \otimes C\right)$ via

$$
(a, \psi): b_{m} \mapsto\left(a(m), b_{m}, \psi\left(b_{m}\right)\right), \quad \text { where } \psi \in \Gamma\left(B^{*} \otimes C\right), \quad a \in \Gamma(A)
$$


In particular, the fibered product $A \times_{M} B$ is a double vector bundle with side bundles $A$ and $B$ and core $M \times 0$.

\subsubsection{Decompositions and lifts}

A decomposition of $(D ; A, B ; M)$ is a diffeomorphism $\mathbb{I}: D \rightarrow \bar{D}=A \times{ }_{M} B \times{ }_{M} C$, as in Definition 2.1; that is, $\mathbb{I}$ is an isomorphism of double vector bundles over the identity maps on $A, B$ and $C$.

Given an injective morphism of double vector bundles $\Sigma: A \times_{M} B \hookrightarrow D$ over the identity on the sides $A$ and $B$, define a decomposition of $D$ by $\mathbb{I}\left(a_{m}, b_{m}, c_{m}\right)=$ $\Sigma\left(a_{m}, b_{m}\right)+{ }_{B}\left(\tilde{0}_{b_{m}}+{ }_{A} \overline{c_{m}}\right)$. Decompositions of $D$ are in bijective correspondence with such morphisms $\Sigma$. We will often refer to $\Sigma$ as a decomposition of $D$.

Decompositions of $D$ are also equivalent to splittings $\sigma_{A}$ of the short exact sequence of $C^{\infty}(M)$-modules

$$
0 \longrightarrow \Gamma\left(B^{*} \otimes C\right) \hookrightarrow \Gamma_{B}^{\ell}(D) \longrightarrow \Gamma(A) \longrightarrow 0,
$$

where the third map is the map that sends a linear section $(\xi, a)$ to its base section $a \in \Gamma(A)$. The splitting $\sigma_{A}$ will be called a lift. Given a decomposition, the lift $\sigma_{A}: \Gamma(A) \rightarrow \Gamma_{B}^{\ell}(D)$ is given by $\sigma_{A}(a)\left(b_{m}\right)=\Sigma\left(a(m), b_{m}\right)=\mathbb{I}^{-1}\left(a(m), b_{m}, 0_{m}\right)$ for all $a \in \Gamma(A)$ and $b_{m} \in B$.

In the case of the tangent double of a vector bundle $E \rightarrow M$, the lift from vector fields on $M$ to vector fields on $E$ (see Sect. 2.1.2) would be the horizontal lift corresponding to a connection. We avoid the word 'horizontal' here since 'horizontal' and 'vertical' refer to the two structures on $D$.

By the symmetry of a decomposition, this implies that a lift $\sigma_{A}: \Gamma(A) \rightarrow \Gamma_{B}^{\ell}(D)$ is equivalent to a lift $\sigma_{B}: \Gamma(B) \rightarrow \Gamma_{A}^{\ell}(D)$. Given a lift $\sigma_{A}: \Gamma(A) \rightarrow \Gamma_{B}^{\ell}(D)$, the corresponding lift $\sigma_{B}: \Gamma(B) \rightarrow \Gamma_{A}^{\ell}(D)$ is given by $\sigma_{B}(b)(a(m))=\sigma_{A}(a)(b(m))$ for all $a \in \Gamma(A), b \in \Gamma(B)$.

Note finally that two decompositions of $D$ differ by a section $\phi_{12}$ of $A^{*} \otimes B^{*} \otimes C \simeq$ $\operatorname{Hom}\left(A, B^{*} \otimes C\right) \simeq \operatorname{Hom}\left(B, A^{*} \otimes C\right)$ in the following sense. For each $a \in \Gamma(A)$ the difference $\sigma_{A}^{1}(a)-{ }_{B} \sigma_{A}^{2}(a)$ of lifts is the core-linear section defined by $\phi_{12}(a) \in$ $\Gamma\left(B^{*} \otimes C\right)$. By symmetry, $\sigma_{B}^{1}(b)-{ }_{A} \sigma_{B}^{2}(b)=\widehat{\phi_{12}(b)}$ for each $b \in \Gamma(B)$.

\subsubsection{The tangent double vector bundle of a vector bundle}

Let $q_{E}: E \rightarrow M$ be a vector bundle. Then the tangent bundle $T E$ has two vector bundle structures; one as the tangent bundle of the manifold $E$, and the second as a vector bundle over $T M$. The structure maps of $T E \rightarrow T M$ are the derivatives of the structure maps of $E \rightarrow M$. The space $T E$ is a double vector bundle with core bundle $E \rightarrow M$.

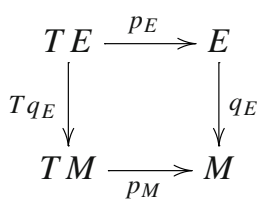


The core vector field corresponding to $e \in \Gamma(E)$ is the vertical lift $e^{\uparrow}: E \rightarrow T E$, i.e. the vector field with flow $\phi: E \times \mathbb{R} \rightarrow E, \phi_{t}\left(e_{m}^{\prime}\right)=e_{m}^{\prime}+t e(m)$. An element of $\Gamma_{E}^{\ell}(T E)=\mathfrak{X}^{\ell}(E)$ is called a linear vector field. It is well-known (see e.g. [15]) that a linear vector field $\xi \in \mathfrak{X}^{l}(E)$ covering $X \in \mathfrak{X}(M)$ corresponds to a derivation $D^{*}: \Gamma\left(E^{*}\right) \rightarrow \Gamma\left(E^{*}\right)$ over $X \in \mathfrak{X}(M)$, and hence to a derivation $D: \Gamma(E) \rightarrow \Gamma(E)$ over $X \in \mathfrak{X}(M)$ (the dual derivation). The precise correspondence is given by ${ }^{1}$

$$
\xi\left(\ell_{\varepsilon}\right)=\ell_{D^{*}(\varepsilon)} \quad \text { and } \quad \xi\left(q_{E}^{*} f\right)=q_{E}^{*}(X(f))
$$

for all $\varepsilon \in \Gamma\left(E^{*}\right)$ and $f \in C^{\infty}(M)$. Here $\ell_{\varepsilon}$ is the linear function $E \rightarrow \mathbb{R}$ corresponding to $\varepsilon$. We will write $\widehat{D}$ for the linear vector field corresponding in this manner to a derivation $D$ of $\Gamma(E)$. The choice of a decomposition for $(T E ; T M, E ; M)$ is equivalent to the choice of a connection on $E$ : Since a decomposition gives us a linear vector field $\sigma_{T M}(X) \in \mathfrak{X}^{l}(E)$ for each $X$, we can define $\nabla: \mathfrak{X}(M) \times \Gamma(E) \rightarrow \Gamma(E)$ by $\sigma_{T M}(X)=\widehat{\nabla_{X}}$ for all $X \in \mathfrak{X}(M)$. Conversely, a connection $\nabla: \mathfrak{X}(M) \times \Gamma(E) \rightarrow$ $\Gamma(E)$ defines a lift $\sigma_{T M}^{\nabla}$ for $(T E ; T M, E ; M)$ and a decomposition of $E$.

We recall as well the relation between the connection and the Lie bracket of vector fields on $E$. Given $\nabla$, it is easy to see using the equalities in (3) that, writing $\sigma$ for $\sigma_{T M}^{\nabla}$ :

$$
[\sigma(X), \sigma(Y)]=\sigma[X, Y]-R_{\nabla}(X, Y)^{\uparrow}, \quad\left[\sigma(X), e^{\uparrow}\right]=\left(\nabla_{X} e\right)^{\uparrow}, \quad\left[e^{\uparrow}, e^{{ }^{\uparrow}}\right]=0,
$$

for all $X, Y \in \mathfrak{X}(M)$ and $e, e^{\prime} \in \Gamma(E)$. That is, the Lie bracket of vector fields on $M$ and the connection encode completely the Lie bracket of vector fields on $E$. One result of this paper is an extension of this description to general double Lie algebroids.

Now let us have a quick look at the other structure on the double vector bundle $T E$. The lift $\sigma_{E}^{\nabla}: \Gamma(E) \rightarrow \Gamma_{T M}^{\ell}(T E)$ is given by

$$
\sigma_{E}^{\nabla}(e)(v)=T e(v)+_{T M}\left(T 0^{E}(v)-{ }_{E} \overline{\nabla_{v} e}\right), \quad v \in T M, \quad e \in \Gamma(E)
$$

\subsubsection{Dualization and lifts}

Recall that double vector bundles can be dualized in two distinct ways. We denote the dual of $D$ as a vector bundle over $A$ by $D * A$ and likewise for $D * B$. The dual $D * A$ is itself a double vector bundle, with side bundles $A$ and $C^{*}$ and core $B^{*}[13,16]$.
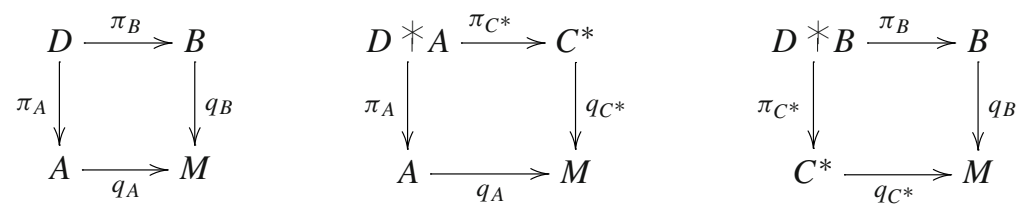

\footnotetext{
1 Since its flow is a flow of vector bundle morphisms, a linear vector field sends linear functions to linear functions and pullbacks to pullbacks.
} 
Note also that by dualizing again $D * B$ over $C^{*}$, we get

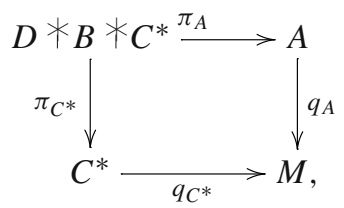

with core $B^{*}$. In the same manner, we get a double vector bundle $D * A * C^{*}$ with sides $B$ and $C^{*}$ and core $A^{*}$.

Recall first of all that the vector bundles $D^{*} B \rightarrow C^{*}$ and $D^{*} A \rightarrow C^{*}$ are, up to a sign, naturally in duality to each other [13]. The pairing

$$
\langle\cdot, \cdot\rangle:(D * A) \times_{C^{*}}(D * B) \rightarrow \mathbb{R}
$$

is defined as follows: for $\Phi \in D * A$ and $\Psi \in D * B$ projecting to the same element $\gamma_{m}$ in $C^{*}$, choose $d \in D$ with $\pi_{A}(d)=\pi_{A}(\Phi)$ and $\pi_{B}(d)=\pi_{B}(\Psi)$. Then $\langle\Phi, d\rangle_{A}-$ $\langle\Psi, d\rangle_{B}$ does not depend on the choice of $d$ and we set $\langle\Phi, \Psi\rangle=\langle\Phi, d\rangle_{A}-\langle\Psi, d\rangle_{B}$.

This implies in particular that $D * A$ is canonically (up to a sign) isomorphic to $D * B * C^{*}$ and $D * B$ is isomorphic to $D * A * C^{*}$. We will use this below.

Each linear section $\xi \in \Gamma_{B}(D)$ over $a \in \Gamma(A)$ induces a linear section $\xi^{\square} \in$ $\Gamma_{C^{*}}^{\ell}\left(D * B * C^{*}\right)$ over $a$. Namely $\xi$ induces a function $\ell_{\xi}: D * B \rightarrow \mathbb{R}$ which is fibrewise-linear over $B$ and, using the definition of the addition in $D^{*} B \rightarrow C^{*}[16$, Equation (7)], it follows that $\ell_{\xi}$ is also linear over $C^{*}$. The corresponding section of $D^{*} B * C^{*} \rightarrow C^{*}$ is denoted $\xi^{\square}[16]$. Thus

$$
\left\langle\xi^{\sqcap}(\gamma), \Psi\right\rangle_{C^{*}}=\ell_{\xi}(\Psi)=\langle\Psi, \xi(b)\rangle_{B}
$$

for $\Psi \in D * B$ such that $\pi_{B}(\Psi)=b$ and $\pi_{C^{*}}(\Psi)=\gamma$.

Given a decomposition $\Sigma: A \times_{M} B \rightarrow D$ of $D$, we get hence a decomposition

$\Sigma^{\star, B}: C^{*} \times_{M} A \rightarrow D * B * C^{*}$, defined by the corresponding lift $\sigma_{A}^{\star, B}: \Gamma(A) \rightarrow$ $\Gamma_{C^{*}}^{\ell}\left(D * B * C^{*}\right)$ :

$$
\sigma_{A}^{\star, B}(a)=\left(\sigma_{A}(a)\right)^{\sqcap}
$$

for all $a \in \Gamma(A)$.

We now use the (canonical up to a sign) isomorphism of $D * A$ with $D * B * C^{*}$ to construct a canonical decomposition of $D * A$ given a decomposition of $D$. We identify $D * A$ with $D * B * C^{*}$ using $-\langle\cdot, \cdot\rangle$. Thus we define the lift $\sigma_{A}^{\star}: \Gamma(A) \rightarrow \Gamma_{C^{*}}^{\ell}(D * A)$ by

$$
\left\langle\sigma_{A}^{\star}(a), \cdot\right\rangle=-\sigma_{A}^{\star, B}(a)
$$

for all $a \in \Gamma(A)$. Note that by (6), this implies that

$$
\left\langle\sigma_{A}^{\star}(a)(\gamma), \sigma_{A}(a)(b)\right\rangle_{A}=0
$$


for all $\gamma \in C^{*}$ and $b \in B$. The choice of sign in (7) is necessary for $\sigma_{A}^{\star}(a)$ to be a linear section of $D^{*} A$ over $a$. To be more explicit, check or recall from [15, Equation (28), p. 352] that $\left\langle\sigma_{A}^{\star}(a), \alpha^{\dagger}\right\rangle=-q_{C^{*}}^{*}\langle\alpha, a\rangle$ for all $\alpha \in \Gamma\left(A^{*}\right)$ (and $\alpha^{\dagger}$ the corresponding core section of $D^{*} B$ over $\left.C^{*}\right)$. But $\left\langle\sigma_{A}^{\star, B}(a), \alpha^{\dagger}\right\rangle_{C^{*}}=q_{C^{*}}^{*}\langle a, \alpha\rangle$ by definition of the pairing of $D * B * C^{*}$ with $D * B$. Hence, without the choice of sign that we make, $\sigma_{A}^{\star}(a)$ would be linear over $-a$, hence not a lift.

By (skew-)symmetry, given the lift $\sigma_{B}: \Gamma(B) \rightarrow \Gamma_{A}^{\ell}(D)$, we identify $D * B$ with $D * A * C^{*}$ using $\langle\cdot, \cdot\rangle$ and define the lift $\sigma_{B}^{\star}: \Gamma(B) \rightarrow \Gamma_{C^{*}}^{\ell}(D * B)$ by $\left\langle\sigma_{B}^{\star}(b), \cdot\right\rangle=$ $\sigma_{B}^{\star}, A(b)$ for all $b \in \Gamma(B)$. (This time, we do not need the minus sign.) As a summary, we have the equations:

$$
\left\langle\sigma_{A}^{\star}(a), \sigma_{B}^{\star}(b)\right\rangle=0, \quad\left\langle\sigma_{A}^{\star}(a), \alpha^{\dagger}\right\rangle=-q_{C^{*}}^{*}\langle\alpha, a\rangle, \quad\left\langle\beta^{\dagger}, \sigma_{B}^{\star}(b)\right\rangle=q_{C^{*}}^{*}\langle\beta, b\rangle,
$$

for all $a \in \Gamma(A), b \in \Gamma(B), \alpha \in \Gamma\left(A^{*}\right)$ and $\beta \in \Gamma\left(B^{*}\right)$. See also [15, §9.2].

\subsection{LA-vector bundles and double Lie algebroids}

Let $(D ; A, B ; M)$ be a double vector bundle

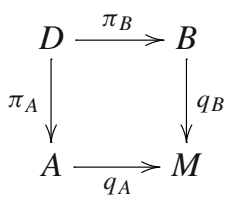

with core $C$. Then $(D ; A, B ; M)$ is a LA-vector bundle [12] if there are Lie algebroid structures on $D \rightarrow B$ and $A \rightarrow M$, such that the vector bundle operations in $D \rightarrow A$ are Lie algebroid morphisms over the corresponding operations in $B \rightarrow M$. As a consequence, the anchor $\Theta_{B}: D \rightarrow T B$ is a morphism of double vector bundles.

An equivalent concept was introduced in [4] with the name VB-algebroid: $(D \rightarrow$ $B ; A \rightarrow M)$ is a $V B$-algebroid if $D \rightarrow B$ is a Lie algebroid, the anchor $\Theta_{D}: D \rightarrow T B$ is a bundle morphism over $\rho_{A}: A \rightarrow T M$ and the Lie bracket is linear:

$\left[\Gamma_{B}^{\ell}(D), \Gamma_{B}^{\ell}(D)\right] \subset \Gamma_{B}^{\ell}(D), \quad\left[\Gamma_{B}^{\ell}(D), \Gamma_{B}^{c}(D)\right] \subset \Gamma_{B}^{c}(D), \quad\left[\Gamma_{B}^{c}(D), \Gamma_{B}^{c}(D)\right]=0$.

The vector bundle $A \rightarrow M$ is then also a Lie algebroid, with anchor $\rho_{A}$ and bracket defined as follows: if $\xi_{1}, \xi_{2} \in \Gamma_{B}^{\ell}(D)$ are linear over $a_{1}, a_{2} \in \Gamma(A)$, then the bracket $\left[\xi_{1}, \xi_{2}\right]$ is linear over $\left[a_{1}, a_{2}\right]$.

Example 2.5 For a vector bundle $E$ on $M$, the tangent double vector bundle $(T E ; E, T M ; M)$ has an LA-vector bundle structure $(T E \rightarrow E, T M \rightarrow M)$ with respect to the standard Lie algebroid structures on $T E \rightarrow E$ and $T M \rightarrow M$.

If $D$ is an LA-vector bundle with Lie algebroid structures on $D \rightarrow B$ and $A \rightarrow M$ the dual vector bundle $D * B \rightarrow B$ has a Lie-Poisson structure (a linear Poisson structure), and the structure on $D^{*} B$ is also Lie-Poisson with respect to $D{ }^{*} B \rightarrow C^{*}$ 
[16, 3.4]. Dualizing this bundle gives a Lie algebroid structure on $D{ }^{*} B{ }^{*} C^{*} \rightarrow C^{*}$. This equips the double vector bundle $\left(D * B * C^{*} ; C^{*}, A ; M\right)$ with a LA-vector bundle structure. Using the isomorphism defined by $-\langle\cdot, \cdot\rangle$, the double vector bundle $\left(D * A ; A, C^{*} ; M\right)$ also has a LA-vector bundle structure.

Definition 2.6 [16] A double Lie algebroid is a double vector bundle $(D ; A, B ; M)$ with core denoted $C$, and with Lie algebroid structures on each of $A \rightarrow M, B \rightarrow M$, $D \rightarrow A$ and $D \rightarrow B$ such that each pair of parallel Lie algebroids gives $D$ the structure of an LA-vector bundle, and such that $\left(D * A * C^{*}, D * B * C^{*}\right)$ with the induced Lie algebroid structures on base $C^{*}$ as defined above, is a Lie bialgebroid.

Equivalently, $D$ is a double Lie algebroid if the pair $(D * A, D * B)$ with the induced Lie algebroid structures on base $C^{*}$ and the pairing $\langle\cdot, \cdot\rangle$, is a Lie bialgebroid. One aim of this paper is to reformulate this definition in terms of specific classes of sections, so as to allow the user to bypass frequent use of the duality of doubles; see Theorem 3.6.

\subsection{Representations up to homotopy and LA-vector bundles}

Let $A \rightarrow M$ be a Lie algebroid and consider an $A$-connection $\nabla$ on a vector bundle $E \rightarrow M$. Then the space $\Omega^{\bullet}(A, E)$ of $E$-valued Lie algebroid forms has an induced operator $\mathbf{d}_{\nabla}$ given by the É. Cartan formula:

$$
\begin{aligned}
\mathbf{d}_{\nabla} \omega\left(a_{1}, \ldots, a_{k+1}\right)= & \sum_{i<j}(-1)^{i+j} \omega\left(\left[a_{i}, a_{j}\right], a_{1}, \ldots, \hat{a}_{i}, \ldots, \hat{a}_{j}, \ldots, a_{k+1}\right) \\
& +\sum_{i}(-1)^{i+1} \nabla_{a_{i}}\left(\omega\left(a_{1}, \ldots, \hat{a}_{i}, \ldots, a_{k+1}\right)\right)
\end{aligned}
$$

for all $\omega \in \Omega^{k}(A, E)$ and $a_{1}, \ldots, a_{k+1} \in \Gamma(A)$.

Let now $\mathcal{E}=\bigoplus_{k \in \mathbb{Z}} E_{k}$ be a graded vector bundle. Consider the space $\Omega^{\bullet}(A, \mathcal{E})$ with grading given by

$$
\Omega^{\bullet}(A, \mathcal{E})^{k}=\bigoplus_{i+j=k} \Omega^{i}\left(A, E_{j}\right)
$$

Definition 2.7 [1] A representation up to homotopy of $A$ on $\mathcal{E}$ is a map $\mathcal{D}: \Omega^{\bullet}(A, \mathcal{E})$ $\rightarrow \Omega^{\bullet}(A, \mathcal{E})$ with total degree 1 and such that $\mathcal{D}^{2}=0$ and

$\mathcal{D}(\alpha \wedge \omega)=\mathbf{d}_{A} \alpha \wedge \omega+(-1)^{|\alpha|} \alpha \wedge \mathcal{D}(\omega), \quad$ for $\alpha \in \Gamma\left(\wedge^{\bullet} A^{*}\right), \quad \omega \in \Omega^{\bullet}(A, \mathcal{E})$,

where $\mathbf{d}_{A}: \Gamma\left(\wedge^{\bullet} A^{*}\right) \rightarrow \Gamma\left(\wedge^{\bullet} A^{*}\right)$ is the Lie algebroid differential.

Note that Gracia-Saz and Mehta [4] defined this concept independently and called it a "superrepresentation"; it is related to Quillen's notion of superconnection [22] in the same way that representations of Lie algebroids are related to the general notion of A-connection. 
The representations up to homotopy which we will consider are always on graded vector bundles $\mathcal{E}=E_{0} \oplus E_{1}$ concentrated on degrees 0 and 1; so-called 2-term graded vector bundles. In this case the data of [1] can be reformulated as follows (see $[1,4]$ ).

Definition 2.8 Let $A$ be a Lie algebroid and let $\mathcal{E}=E_{0} \oplus E_{1}$ be a 2-term graded vector bundle on the same base. Then a 2-term representation up to homotopy or, for brevity, a 2-representation of $A$ on $\mathcal{E}$, consists of

(i) a map $\partial: E_{0} \rightarrow E_{1}$,

(ii) two $A$-connections, $\nabla^{0}$ and $\nabla^{1}$ on $E_{0}$ and $E_{1}$, respectively, such that $\partial \circ \nabla^{0}=$ $\nabla^{1} \circ \partial$

(iii) an element $R \in \Omega^{2}\left(A, \operatorname{Hom}\left(E_{1}, E_{0}\right)\right)$ such that $R_{\nabla^{0}}=R \circ \partial, R_{\nabla^{1}}=\partial \circ R$ and $\mathbf{d}_{\nabla \text { Hom }} R=0$, where $\nabla^{\text {Hom }}$ is the connection induced on $\operatorname{Hom}\left(E_{1}, E_{0}\right)$ by $\nabla^{0}$ and $\nabla^{1}$.

Proposition 2.9 Let $A$ be a Lie algebroid and let $\mathcal{E}=E_{0} \oplus E_{1}$ be a 2-term graded vector bundle on the same base. Given a 2-representation of $A$ on $\mathcal{E}$, define $\mathcal{D}: \Gamma E^{0} \rightarrow$ $\Omega^{1}\left(A, E^{0}\right) \oplus \Gamma E^{1}$ by $\mathcal{D}\left(e^{0}\right)=\nabla^{0}\left(e^{0}\right) \oplus \partial\left(e^{0}\right)$ and define $\mathcal{D}: \Omega^{1}\left(A, E^{0}\right) \oplus \Gamma E^{1} \rightarrow$ $\Omega^{2}\left(A, E^{0}\right) \oplus \Omega^{1}\left(A, E^{1}\right)$ by

$$
\mathcal{D}\left(\omega \oplus e^{1}\right)=\left(-R\left(e^{1}\right)-d_{\nabla^{0}}(\omega)\right) \oplus\left(\partial(\omega)-\nabla^{1}\left(e^{1}\right)\right) .
$$

Then $\mathcal{D}$ extends to a representation up to homotopy of $A$ on $\mathcal{E}$. This defines a bijective correspondence between 2-representations of $A$ on $\mathcal{E}$ and representations up to homotopy of $A$ on $\mathcal{E}$.

Let $(D \rightarrow B ; A \rightarrow M)$ be an LA-vector bundle. Recall that since the anchor $\Theta_{B}$ is linear, it sends a core section $c^{\dagger}, c \in \Gamma(C)$ to a vertical vector field on $B$. This defines the core-anchor $\partial_{B}: C \rightarrow B$ given by, $\Theta_{B}\left(c^{\dagger}\right)=\left(\partial_{B} c\right)^{\uparrow}$ for all $c \in \Gamma(C)$ (see [11]).

Choose further a decomposition $\Sigma: A \times_{M} B \rightarrow D$. Since the anchor of a linear section is linear, for each $a \in \Gamma(A)$ the vector field $\Theta_{B}\left(\sigma_{A}(a)\right)$ defines a derivation of $\Gamma(B)$ with symbol $\rho(a)$ (see Sect. 2.1.2). This defines a linear connection $\nabla^{A B}: \Gamma(A) \times \Gamma(B) \rightarrow \Gamma(B):$

$$
\Theta_{B}\left(\sigma_{A}(a)\right)=\widehat{\nabla_{a}^{A B}}
$$

for all $a \in \Gamma(A)$. Since the bracket of a linear section with a core section is again a core section, we find a linear connection $\nabla^{A C}: \Gamma(A) \times \Gamma(C) \rightarrow \Gamma(C)$ such that

$$
\left[\sigma_{A}(a), c^{\dagger}\right]=\left(\nabla_{a}^{A C} c\right)^{\dagger}
$$

for all $c \in \Gamma(C)$ and $a \in \Gamma(A)$. The difference $\sigma_{A}\left[a_{1}, a_{2}\right]-\left[\sigma_{A}\left(a_{1}\right), \sigma_{A}\left(a_{2}\right)\right]$ is a core-linear section for all $a_{1}, a_{2} \in \Gamma(A)$. This defines a vector valued Lie algebroid form $R \in \Omega^{2}(A, \operatorname{Hom}(B, C))$ such that

$$
\left[\sigma_{A}\left(a_{1}\right), \sigma_{A}\left(a_{2}\right)\right]=\sigma_{A}\left[a_{1}, a_{2}\right]-\widetilde{R\left(a_{1}, a_{2}\right)}
$$

for all $a_{1}, a_{2} \in \Gamma(A)$. See [4] for more details on these constructions. 
The following theorem is proved in [4].

Theorem 2.10 Let $(D \rightarrow B ; A \rightarrow M)$ be a LA-vector bundle and choose a decomposition $\Sigma: A \times_{M} B \rightarrow D$. The triple $\left(\nabla^{A B}, \nabla^{A C}, R\right)$ defined as above is a 2-representation of $A$ on the complex $\partial_{B}: C \rightarrow B$.

Conversely, let $(D ; A, B ; M)$ be a double vector bundle such that $A$ has a Lie algebroid structure and choose a decomposition $\Sigma: A \times{ }_{M} B \rightarrow D$. Then if $\left(\nabla^{A B}, \nabla^{A C}, R\right)$ is a 2-representation of $A$ on a complex $\partial_{B}: C \rightarrow B$, then the four equations above define a LA-vector bundle structure on $(D \rightarrow B ; A \rightarrow M)$.

The following formulas for the brackets of linear and core sections with core-linear sections will be very useful in the proof of our main theorem. In the situation of the previous theorem, we have

$$
\left[\sigma_{A}(a), \tilde{\phi}\right]=\widetilde{\nabla_{a}^{\mathrm{Hom}} \phi}
$$

and

$$
\left[c^{\dagger}, \tilde{\phi}\right]=\left(\phi\left(\partial_{B} c\right)\right)^{\dagger}
$$

for all $a \in \Gamma(A), \phi \in \Gamma(\operatorname{Hom}(B, C))$ and $c \in \Gamma(C)$. To see this, write $\phi$ as $\sum f_{i j} \cdot \beta_{i} \cdot c_{j}$ with $f_{i j} \in C^{\infty}(M), \beta_{i} \in \Gamma\left(B^{*}\right)$ and $c_{j} \in \Gamma(C)$. Then $\widetilde{\phi}=\sum q_{B}^{*} f_{i j} \cdot \ell_{\beta_{i}} \cdot c_{j}^{\dagger}$ and one can use the formulas in Theorem 2.10 and the Leibniz rule to compute the brackets with $\sigma_{A}(a)$ and $c^{\dagger}$.

Note that (10) and (11) can also be proved by diagrammatic methods.

Example 2.11 Choose a linear connection $\nabla: \mathfrak{X}(M) \times \Gamma(E) \rightarrow \Gamma(E)$ and consider the corresponding decomposition $\Sigma^{\nabla}$ of $T E$ as in Sect. 2.1.2. The description of the Lie bracket of vector fields in (4) shows that the 2-representation induced by $\Sigma^{\nabla}$ is the 2-representation of $T M$ on $\operatorname{Id}_{E}: E \rightarrow E$ given by $\left(\nabla, \nabla, R_{\nabla}\right)$.

Remark 2.12 Let $\Sigma_{1}, \Sigma_{2}: A \times_{M} B \rightarrow D$ be two decompositions of a LA-vector bundle $(D \rightarrow B, A \rightarrow M)$, and write $\phi_{12}$ for the section of $A^{*} \otimes B^{*} \otimes C$ defined in Sect. 2.1.1. Regarding $\phi_{12}$ as an element of $\operatorname{Hom}\left(A, B^{*} \otimes C\right)$ and as an element of $\operatorname{Hom}\left(B, A^{*} \otimes C\right)$, the two corresponding 2-representations are related by the following identities [4].

$$
\nabla_{a}^{B, 2}=\nabla_{a}^{B, 1}+\partial_{B} \circ \phi_{12}(a), \quad \nabla_{a}^{C, 2}=\nabla_{a}^{C, 1}+\phi_{12}(a) \circ \partial_{B}
$$

and

$$
\begin{aligned}
& R^{2}\left(a_{1}, a_{2}\right)=R^{1}\left(a_{1}, a_{2}\right)+\left(\mathbf{d}_{\nabla \operatorname{Hom}(B, C)} \phi_{12}\right)\left(a_{1}, a_{2}\right)+\phi_{12}\left(a_{1}\right) \partial_{B} \phi_{12}\left(a_{2}\right) \\
& \quad-\phi_{12}\left(a_{2}\right) \partial_{B} \phi_{12}\left(a_{1}\right)
\end{aligned}
$$

for all $a, a_{1}, a_{2} \in \Gamma(A)$.

\subsubsection{Dualization and 2-representations}

Let $(D ; A, B ; M)$ be a LA-vector bundle with Lie algebroid structures on $D \rightarrow B$ and $A \rightarrow M$. Let $\Sigma: A \times_{M} B \rightarrow D$ be a decomposition of $D$ and denote by $\left(\nabla^{B}, \nabla^{C}, R\right)$ 
the 2-representation of the Lie algebroid $A$ on $\partial_{B}: C \rightarrow B$. We have seen above that $\left(D^{*} A ; A, C^{*} ; M\right)$ has an induced LA-vector bundle structure, and we have shown that the decomposition $\Sigma$ induces a natural decomposition $\Sigma^{\star}: A \times_{M} C^{*} \rightarrow D * B$ of $D^{*} A$. The 2-representation of $A$ that is associated to this decomposition is then $\left(\nabla^{C^{*}}, \nabla^{B^{*}},-R^{*}\right)$ on the complex $\partial_{B}^{*}: B^{*} \rightarrow C^{*}$. This is easy to verify, and proved in the appendix ${ }^{2}$ of [2]. One only needs to recall for the proof that, by construction, $\ell_{\sigma_{A}^{\star}(a)}$ equals $\ell_{\sigma_{A}(a)}$ as a function on $D * B$.

\subsubsection{The tangent of a Lie algebroid}

Let $(A \rightarrow M, \rho,[\cdot, \cdot])$ be a Lie algebroid. Then the tangent $T A \rightarrow T M$ has a Lie algebroid structure with bracket defined by $\left[T a_{1}, T a_{2}\right]=T\left[a_{1}, a_{2}\right],\left[T a_{1}, a_{2}^{\dagger}\right]=$ $\left[a_{1}, a_{2}\right]^{\dagger}$ and $\left[a_{1}^{\dagger}, a_{2}^{\dagger}\right]=0$ for all $a_{1}, a_{2} \in \Gamma(A)$. The anchor of $T a$ is $[\widehat{\rho(a), \cdot} \cdot] \in$ $\mathfrak{X}(T M)$ and the anchor of $a^{\dagger}$ is $\rho(a)^{\uparrow}$ for all $a \in \Gamma(A)$. This defines a LA-vector bundle structure $(T A \rightarrow T M ; A \rightarrow M)$ on $(T A ; T M, A ; M)$.

Given a $T M$-connection on $A$, and so a decomposition $\Sigma^{\nabla}$ of $T A$ as in Section 2.1.2, the 2-representation of $A$ on $\rho: A \rightarrow T M$ encoding the LA-vector bundle is $\left(\nabla^{\text {bas }}, \nabla^{\text {bas }}, R_{\nabla}^{\text {bas }}\right)$, where the connections are defined by

$$
\nabla^{\text {bas }}: \Gamma(A) \times \mathfrak{X}(M) \rightarrow \mathfrak{X}(M), \quad \nabla_{a}^{\text {bas }} X=[\rho(a), X]+\rho\left(\nabla_{X} a\right),
$$

and

$$
\nabla^{\text {bas }}: \Gamma(A) \times \Gamma(A) \rightarrow \Gamma(A), \quad \nabla_{a_{1}}^{\text {bas }} a_{2}=\left[a_{1}, a_{2}\right]+\nabla_{\rho\left(a_{2}\right)} a_{1},
$$

with $R_{\nabla}^{\text {bas }} \in \Omega^{2}(A, \operatorname{Hom}(T M, A))$ given by

$R_{\nabla}^{\mathrm{bas}}\left(a_{1}, a_{2}\right) X=-\nabla_{X}\left[a_{1}, a_{2}\right]+\left[\nabla_{X} a_{1}, a_{2}\right]+\left[a_{1}, \nabla_{X} a_{2}\right]+\nabla_{\nabla_{a_{2}}^{\mathrm{bas}} X} a_{1}-\nabla_{\nabla_{a_{1}}^{\mathrm{bas}} X} a_{2}$

for all $X \in \mathfrak{X}(M), a, a_{1}, a_{2} \in \Gamma(A)$.

\section{Main theorem and examples}

We define in this section the notion of matched pair of 2-representations. We then state our main result, Theorem 3.6: a double vector bundle endowed with two LA-vector bundle structures (one horizontal, one vertical) is a double Lie algebroid if and only if, for each decomposition, the two induced 2-representations form a matched pair.

In the second part of this section, we work out the example of the structures on the tangent double vector bundle of a Lie algebroid.

\footnotetext{
2 The construction of the "dual" decomposition of $D * A$, given a decomposition of $D$, is done in [2] by dualizing the decomposition and taking its inverse. The resulting decomposition of $D{ }^{*} A$ is the same.
} 


\subsection{Matched pairs of 2-representations and main result}

Definition 3.1 Let $\left(A \rightarrow M, \rho_{A},[\cdot, \cdot]\right)$ and and $\left(B \rightarrow M, \rho_{B},[\cdot, \cdot]\right)$ be two Lie algebroids and assume that $A$ acts on $\partial_{B}: C \rightarrow B$ up to homotopy via $\left(\nabla^{A B}, \nabla^{A C}, R_{A}\right)$ and $B$ acts on $\partial_{A}: C \rightarrow A$ up to homotopy via $\left(\nabla^{B A}, \nabla^{B C}, R_{B}\right)$. Then we say that the two 2-representations form a matched pair if the following hold: ${ }^{3}$

(M1) $\nabla_{\partial_{A} c_{1}} c_{2}-\nabla_{\partial_{B} c_{2}} c_{1}=-\nabla_{\partial_{A} c_{2}} c_{1}+\nabla_{\partial_{B} c_{1}} c_{2}$,

(M2) $\left[a, \partial_{A} c\right]=\partial_{A}\left(\nabla_{a} c\right)-\nabla_{\partial_{B} c} a$,

(M3) $\left[b, \partial_{B} c\right]=\partial_{B}\left(\nabla_{b} c\right)-\nabla_{\partial_{A} c} b$,

(M4) $\nabla_{b} \nabla_{a} c-\nabla_{a} \nabla_{b} c-\nabla_{\nabla_{b}} c+\nabla_{\nabla_{a} b} c=R_{B}\left(b, \partial_{B} c\right) a-R_{A}\left(a, \partial_{A} c\right) b$,

(M5) $\partial_{A}\left(R_{A}\left(a_{1}, a_{2}\right) b\right)=-\nabla_{b}\left[a_{1}, a_{2}\right]+\left[\nabla_{b} a_{1}, a_{2}\right]+\left[a_{1}, \nabla_{b} a_{2}\right]+\nabla_{\nabla_{a_{2}}} a_{1}$

$$
-\nabla_{a_{1} b} a_{2}
$$

(M6) $\partial_{B}\left(R_{B}\left(b_{1}, b_{2}\right) a\right)=-\nabla_{a}\left[b_{1}, b_{2}\right]+\left[\nabla_{a} b_{1}, b_{2}\right]+\left[b_{1}, \nabla_{a} b_{2}\right]+\nabla_{\nabla_{b_{2}} a} b_{1}$

$$
-\nabla_{\nabla_{b_{1}} a} b_{2}
$$

for all $a, a_{1}, a_{2} \in \Gamma(A), b, b_{1}, b_{2} \in \Gamma(B)$ and $c, c_{1}, c_{2} \in \Gamma(C)$, and

(M7) $\mathbf{d}_{\nabla^{A}} R_{B}=\mathbf{d}_{\nabla^{B}} R_{A} \in \Omega^{2}\left(A, \wedge^{2} B^{*} \otimes C\right)=\Omega^{2}\left(B, \wedge^{2} A^{*} \otimes C\right)$, where $R_{B}$ is seen as an element of $\Omega^{1}\left(A, \wedge^{2} B^{*} \otimes C\right)$ and $R_{A}$ as an element of $\Omega^{1}\left(B, \wedge^{2} A^{*} \otimes C\right)$.

Remark 3.2 The conditions in Definition 3.1 imply that

$$
\rho_{A} \circ \partial_{A}=\rho_{B} \circ \partial_{B}
$$

Specifically, if $A$ has nonzero rank, then (12) can be obtained from (M2) by replacing $a$ with $f a$ for $f \in C^{\infty}(M)$. If $B$ has nonzero rank, then (12) can similarly be obtained from (M3). If both $A$ and $B$ have rank zero, then it is trivially satisfied.

Remark 3.3 The conditions in Definition 3.1 also imply that

$$
\left[\rho_{A}(a), \rho_{B}(b)\right]=\rho_{B}\left(\nabla_{a} b\right)-\rho_{A}\left(\nabla_{b} a\right)
$$

for all $a \in \Gamma(A)$ and $b \in \Gamma(B)$. Specifically, if $A$ has nonzero rank, then (M0) can be obtained from (M5) by replacing $a_{2}$ with $f a_{2}$ for $f \in C^{\infty}(M)$. If $B$ has nonzero rank, then (M0) can be similarly obtained from (M6). If both $A$ and $B$ have rank zero, then it is trivially satisfied.

Remark 3.4 Note that if $C$ is trivial, then $\partial_{A}, \partial_{B}, R_{A}, R_{B}$ and $\nabla^{A C}, \nabla^{B C}$ are trivial. In that case, equations (M1)-(M4), (M7) and the left hand sides of (M5) and (M6) vanish. Bearing in mind that (M5)-(M6) imply (M0), we find the definition of a matched pair of representations of Lie algebroids $[10,18]$. In particular we find that (M0) is in fact redundant in the definition of a matched pair of representations of Lie algebroids.

\footnotetext{
3 For the sake of simplicity, from now on we usually write $\nabla$ for all four connections. It is always clear from the arguments which connection is meant. We write $\nabla^{A}$ for the $A$-connection induced by $\nabla^{A B}$ and $\nabla^{A C}$ on $\wedge^{2} B^{*} \otimes C$ and $\nabla^{B}$ for the $B$-connection induced on $\wedge^{2} A^{*} \otimes C$.
} 
Remark 3.5 The vector bundle $C$ inherits a Lie algebroid structure with anchor $\rho_{C}:=$ $\rho_{A} \circ \partial_{A}=\rho_{B} \circ \partial_{B}$ and with bracket given by $\left[c_{1}, c_{2}\right]=\nabla_{\partial_{A} c_{1}} c_{2}-\nabla_{\partial_{B} c_{2}} c_{1}$ for all $c_{1}, c_{2} \in \Gamma(C)$. Using Remark 2.12 one can see that the Lie algebroid structure on $C$ does not depend on the choice of splitting.

The proof of the Jacobi identity is not completely straightforward; it follows from (M2), (M3) and (M4). A detailed proof of a more general result, but with the same type of computation, is given in [7, Theorem 7.7]. Note that (M2) together with $\partial_{A} \circ \nabla^{B C}=$ $\nabla^{B A} \circ \partial_{A}$ (Equation (ii) in Definition 2.8) shows that $\partial_{A}: C \rightarrow A$ is a Lie algebroid morphism. In the same manner, (M3) together with $\partial_{B} \circ \nabla^{A C}=\nabla^{A B} \circ \partial_{B}$ shows that $\partial_{B}: C \rightarrow B$ is a Lie algebroid morphism.

The Lie algebroid structure on $C$ was defined intrinsically in [16]. Referring to Definition 2.6, the Lie bialgebroid ( $D * A * C^{*}, D * B * C^{*}$ ) induces a Poisson structure (natural up to sign) on its base $C^{*}$; this Poisson structure is linear [16, §4] and so induces a Lie algebroid structure on $C$. As with the specific formula for the bracket above, the sign of the Poisson structure is determined by the requirement that $\partial_{A}$ and $\partial_{B}$ be morphisms of Lie algebroids.

Theorem 3.6 is our main result. The proof is in Sect. 4 .

Theorem 3.6 Let $(D ; A, B ; M)$ be a double vector bundle with LA-vector bundle structures on both $(D \rightarrow A, B \rightarrow M)$ and $(D \rightarrow B, A \rightarrow M)$. Choose a decomposition $\Sigma$ of $D$ and let $\mathcal{D}_{A}$ and $\mathcal{D}_{B}$ be the two 2-representations defined by the lifts $\sigma_{A}$ and $\sigma_{B}$. Then $(D ; A, B ; M)$ is a double Lie algebroid if and only if the two 2-representations form a matched pair.

Remark 3.7 An immediate consequence of Theorem 3.6 is that, if $\mathcal{D}_{A}$ and $\mathcal{D}_{B}$ are 2-representations that form a matched pair, and $\mathcal{D}_{A}^{\prime}$ and $\mathcal{D}_{B}^{\prime}$ are the 2-representations obtained by the transformation in Remark 2.12 , then $\mathcal{D}_{A}^{\prime}$ and $\mathcal{D}_{B}^{\prime}$ will also form a matched pair. In other words, equations (M1) to (M7) are invariant under the transformation in Remark 2.12,

Remark 3.8 Given a matched pair of representations of Lie algebroids $A$ and $B$ on the same base $M$, the direct sum vector bundle $A \oplus B$ has a Lie algebroid structure, the bicrossproduct Lie algebroid, denoted $A \bowtie B[10,18]$. The matched pair structure also induces on the decomposed double vector bundle $A \times_{M} B$ a double Lie algebroid structure and, conversely, any vacant double Lie algebroid (that is, a double Lie algebroid for which the core is zero) arises from a matched pair of Lie algebroids in this way $[16, \S 6]$.

\subsection{Comparison with the equations of Voronov}

In [26] Th. Voronov extended the notion of double Lie algebroid to supergeometry and thereby gave an exceptionally elegant reformulation of the original definition. A vector bundle $A$ may be characterized as a Lie algebroid if its parity-reversion $\Pi A$ has a vector field $Q$ of degree +1 which is homological in the sense that $Q^{2}=0[24,25]$.

Consider now a supermanifold $D$ with a double vector structure $(D ; A, B ; M)$. Write $\Pi_{A} D$ and $\Pi_{B} D$ for the parity reversions and let $Q_{A}$ and $Q_{B}$ be homological 
vector fields on $\Pi_{A} D$ and $\Pi_{B} D$ which have degrees $(1,0)$ and $(0,1)$. It follows that $Q_{A}$ and $Q_{B}$ induce Lie algebroid structures on $\Pi A$ and $\Pi B$. Then [26] defines $D$ to be a (super) double Lie algebroid if $\left[Q_{A}, Q_{B}\right]=0$.

Most of the calculations in [26] are carried out in terms of coordinates. Equations (47) and (48) of [26] give coordinate descriptions of the homological vector fields that are equivalent to the two Lie algebroid structures $D^{*} A \rightarrow C^{*}$ and $D * B \rightarrow C^{*}$.

The main work of [26] is to establish an equivalence between the Lie bialgebroid condition in the definition of a double Lie algebroid (Condition III in [26]) and the commutativity condition which we refer to briefly as $\left[Q_{A}, Q_{B}\right]=0$. (In fact $Q_{A}$ and $Q_{B}$ are defined on different bundles, but admit"parity reversions" to $\Pi^{2} D=$ $\Pi_{A} \Pi_{B} D \cong \Pi_{B} \Pi_{A} D$ and it is these parity reversions which must commute.)

This is expressed in terms of eight coordinate equations (50)-(57). We now relate these to our equations (M1) to (M7) and (M0).

A choice of coordinates as in [26] constitutes a local choice of decomposition of $D$, and yields local decompositions of the duals $D * A$ and $D * B$. By Remark 3.7, it is in fact enough to check (M1) to (M7) in local decompositions. As in [26] the letters $\xi_{i}$ denote basis sections for $A$ and $\xi^{i}$ denote the dual basis sections for $A^{*}$. The letters $\eta_{\alpha}$ denote basis sections for $B$ and $\eta^{\alpha}$ denote the dual basis sections for $B^{*}$. The letters $x_{a}$ denote coordinates on the base manifold $M$. Finally the letters $z_{\mu}$ are basis sections for $C$ and $z^{\mu}$ are the dual basis sections for $C^{*}$.

In what follows, we refer to equations from [26] with an initial ' $V$ '. The coefficients in (V 47) can be formulated in our terms as

$$
\begin{aligned}
Q_{\alpha}^{a} & =\rho_{B}\left(\eta_{\alpha}\right)\left(x_{a}\right), \quad Q_{i \alpha}^{j}=-\left\langle\xi^{j}, \nabla_{\eta_{\alpha}} \xi_{i}\right\rangle, \quad Q_{\mu}^{j}=\left\langle\partial_{A}^{*} \xi^{j}, z_{\mu}\right\rangle, \\
Q_{i \beta \alpha}^{\lambda} & =\left\langle R\left(\eta_{\alpha}, \eta_{\beta}\right) \xi_{i}, z^{\lambda}\right\rangle, \quad Q_{\beta \alpha}^{\gamma}=\left\langle\left[\eta_{\beta}, \eta_{\alpha}\right]_{B}, \eta^{\gamma}\right\rangle, \quad Q_{\mu \alpha}^{\lambda}=-\left\langle z^{\lambda}, \nabla_{\eta_{\alpha}} z_{\mu}\right\rangle,
\end{aligned}
$$

where $R, \nabla$ and $\partial$ refer to the 2-representations induced by the local decomposition. We believe that the term $\xi_{i} \eta^{\alpha} Q_{i \alpha}^{j}$ in (V 47) should have a minus sign for consistency with (V 26) and (V 28).

Similarly the coefficients in (V 48) are

$$
\begin{aligned}
Q_{i}^{a} & =\rho_{A}\left(\xi_{i}\right)\left(x_{a}\right), \quad Q_{\alpha i}^{\beta}=-\left\langle\eta^{\beta}, \nabla_{\xi_{i}} \eta_{\alpha}\right\rangle, \quad Q_{\mu}^{\beta}=\left\langle\partial_{B}^{*} \eta^{\beta}, z_{\mu}\right\rangle, \\
Q_{\alpha j i}^{\lambda} & =\left\langle R\left(\xi_{i}, \xi_{j}\right) \eta_{\alpha}, z^{\lambda}\right\rangle, \quad Q_{j i}^{k}=\left\langle\left[\xi_{j}, \xi_{i}\right]_{A}, \xi^{k}\right\rangle, \quad Q_{\mu i}^{\lambda}=-\left\langle z^{\lambda}, \nabla_{\xi_{i}} z_{\mu}\right\rangle .
\end{aligned}
$$

We now describe briefly how Voronov's nine equations (V 50) to (V 58) correspond to our seven equations (M1)-(M7) together with (12) and (M0). We treat two equations in detail and state the remaining correspondences, leaving details to the reader.

Equation (V 50): $Q_{\alpha}^{a} Q_{\mu}^{\alpha}-Q_{\mu}^{i} Q_{i}^{a}=0$ is satisfied for all $\mu$ and $a$ if and only if

$$
\rho_{B}\left(\eta_{\alpha}\right)\left(x_{a}\right)\left\langle\partial_{B}^{*} \eta^{\alpha}, z_{\mu}\right\rangle=\rho_{A}\left(\xi_{i}\right)\left(x_{a}\right)\left\langle\partial_{A}^{*} \xi^{i}, z_{\mu}\right\rangle
$$

for all $\mu$ and $a$. But this is

$$
\rho_{B}\left(\left\langle\partial_{B}^{*} \eta^{\alpha}, z_{\mu}\right\rangle \eta_{\alpha}\right)\left(x_{a}\right)=\rho_{A}\left(\left\langle\partial_{A}^{*} \xi^{i}, z_{\mu}\right\rangle \xi_{i}\right)\left(x_{a}\right)
$$


and we have $\left\langle\partial_{B}^{*} \eta^{\alpha}, z_{\mu}\right\rangle \eta_{\alpha}=\left\langle\eta^{\alpha}, \partial_{B} z_{\mu}\right\rangle \eta_{\alpha}=\partial_{B}\left(z_{\mu}\right)$ and $\left\langle\partial_{A}^{*} \xi^{i}, z_{\mu}\right\rangle \xi_{i}=$ $\left\langle\xi^{i}, \partial_{A} z_{\mu}\right\rangle \xi_{i}=\partial_{A}\left(z_{\mu}\right)$ because of the choices of the dual basis $\left\{\xi_{i}\right\}$ and $\left\{\xi^{i}\right\}$, and $\left\{\eta_{\alpha}\right\}$ and $\left\{\eta^{\alpha}\right\}$. Hence, Equation (V 50) now reads $\rho_{A}\left(\partial_{A}\left(z_{\mu}\right)\right)\left(x_{a}\right)=\rho_{B}\left(\partial_{B}\left(z_{\mu}\right)\right)\left(x_{a}\right)$ for all $\mu, a$. Since $x_{a}$ are coordinates on the base manifold and $\left\{z_{\mu}\right\}$ is a basis for $C$, we get $\rho_{A} \circ \partial_{A}=\rho_{B} \circ \partial_{B}$.

Equation (V 51): $0=-Q_{\mu}^{i} Q_{\nu i}^{\lambda}+Q_{\mu}^{\alpha} Q_{\nu \alpha}^{\lambda}-Q_{\nu}^{i} Q_{\mu i}^{\lambda}+Q_{\nu}^{\alpha} Q_{\mu \alpha}^{\lambda}$ for all $\mu, v, \lambda$ is

$$
\begin{aligned}
0= & \left\langle\partial_{A}^{*} \xi^{i}, z_{\mu}\right\rangle\left\langle z^{\lambda}, \nabla_{\xi_{i}} z_{\nu}\right\rangle-\left\langle\partial_{B}^{*} \eta^{\alpha}, z_{\mu}\right\rangle\left\langle z^{\lambda}, \nabla_{\eta_{\alpha}} z_{\nu}\right\rangle \\
& +\left\langle\partial_{A}^{*} \xi^{i}, z_{\nu}\right\rangle\left\langle z^{\lambda}, \nabla_{\xi_{i}} z_{\mu}\right\rangle-\left\langle\partial_{B}^{*} \eta^{\alpha}, z_{\nu}\right\rangle\left\langle z^{\lambda}, \nabla_{\eta_{\alpha}} z_{\mu}\right\rangle
\end{aligned}
$$

for all $\mu, v, \lambda$. As before, this is

$$
\begin{aligned}
0 & =\left\langle z^{\lambda}, \nabla_{\partial_{A} z_{\mu}} z_{\nu}\right\rangle-\left\langle z^{\lambda}, \nabla_{\partial_{B} z_{\mu}} z_{\nu}\right\rangle+\left\langle z^{\lambda}, \nabla_{\partial_{A} z_{\nu}} z_{\mu}\right\rangle-\left\langle z^{\lambda}, \nabla_{\partial_{B} z_{\nu}} z_{\mu}\right\rangle \\
& =\left\langle z^{\lambda}, \nabla_{\partial_{A} z_{\mu}} z_{\nu}-\nabla_{\partial_{B} z_{\mu}} z_{\nu}+\nabla_{\partial_{A} z_{\nu}} z_{\mu}-\nabla_{\partial_{B} z_{\nu}} z_{\mu}\right\rangle
\end{aligned}
$$

for all $\mu, v, \lambda$. This implies $\nabla_{\partial_{A} z_{\mu}} z_{\nu}-\nabla_{\partial_{B} z_{\nu}} z_{\mu}=-\left(\nabla_{\partial_{A} z_{\nu}} z_{\mu}-\nabla_{\partial_{B} z_{\mu}} z_{\nu}\right)$ for all $\mu, v$, which is exactly (M1).

As the reader can see, the proof of the equivalences requires writing out Voronov's equations in terms of the 2-representations (see (13) and (14)), and using the duality of the coordinates. The proof of the remaining equivalences can be done in the same manner. We leave the details to the reader.

Equation (V 52): $Q_{\alpha j}^{\beta} Q_{\beta}^{a}+Q_{j}^{b} \partial_{b} Q_{\alpha}^{a}-Q_{j \alpha}^{i} Q_{i}^{a}=Q_{\alpha}^{b} \partial_{b} Q_{j}^{a}$ is our (M0).

Equation (V 53): $Q_{j}^{a} \partial_{a} Q_{\mu}^{i}+Q_{\mu}^{k} Q_{j k}^{i}-Q_{\mu}^{\alpha} Q_{j \alpha}^{i}=-Q_{\mu j}^{\lambda} Q_{\lambda}^{i}$ is (M2).

\section{Equation (V 54):}

$$
\begin{aligned}
& Q_{\mu}^{i} Q_{\beta j i}^{\lambda}-Q_{\beta j}^{\alpha} Q_{\mu \alpha}^{\lambda}+Q_{\nu j}^{\lambda} Q_{\mu \beta}^{\nu}-Q_{j}^{a} \partial_{a} Q_{\mu \beta}^{\lambda}+Q_{\mu i}^{\lambda} Q_{j \beta}^{i}-Q_{\mu}^{\alpha} Q_{j \beta \alpha}^{\lambda} \\
& =-Q_{\beta}^{a} \partial_{a} Q_{\mu j}^{\lambda}+Q_{\mu j}^{\nu} Q_{\nu \beta}^{\lambda}
\end{aligned}
$$

is (M4).

Equation (V 55): $-Q_{\mu}^{i} Q_{\alpha i}^{\gamma}+Q_{\lambda}^{\gamma} Q_{\mu \alpha}^{\lambda}-Q_{\mu}^{\beta} Q_{\beta \alpha}^{\gamma}=-Q_{\alpha}^{a} \partial_{a} Q_{\mu}^{\gamma}$ is (M3).

\section{Equation (V 56):}

$$
\begin{aligned}
& Q_{\alpha j}^{\beta} Q_{i \beta}^{k}+Q_{j l}^{k} Q_{i \alpha}^{l}+Q_{j}^{a} \partial_{a} Q_{i \alpha}^{k}-Q_{\alpha i}^{\beta} Q_{j \beta}^{k}-Q_{i l}^{k} Q_{j \alpha}^{l}-Q_{i}^{a} \partial_{a} Q_{j \alpha}^{k} \\
& \quad=Q_{\alpha}^{a} \partial_{a} Q_{i j}^{k}-Q_{i j}^{l} Q_{l \alpha}^{k}-Q_{\alpha i j}^{\mu} Q_{\mu}^{k}
\end{aligned}
$$

is (M5), providing the last term in (V 56) is changed to a positive sign. 


\section{Equation (V 57):}

$$
\begin{aligned}
& Q_{\alpha i k}^{\lambda} Q_{j \beta}^{k}-Q_{\beta i k}^{\lambda} Q_{j \alpha}^{k}-Q_{\alpha i}^{\gamma} Q_{j \beta \gamma}^{\lambda}+Q_{\beta i}^{\gamma} Q_{j \alpha \gamma}^{\lambda}+Q_{\mu i}^{\lambda} Q_{j \beta \alpha}^{\mu}-Q_{i}^{a} \partial_{a} Q_{j \beta \alpha}^{\lambda} \\
& -Q_{\alpha j k}^{\lambda} Q_{i \beta}^{k}+Q_{\beta j k}^{\lambda} Q_{i \alpha}^{k}+Q_{\alpha j}^{\gamma} Q_{i \beta \gamma}^{\lambda}-Q_{\beta j}^{\gamma} Q_{i \alpha \gamma}^{\lambda}-Q_{\mu j}^{\lambda} Q_{i \beta \alpha}^{\mu}+Q_{j}^{a} \partial_{a} Q_{i \beta \alpha}^{\lambda} \\
& =-Q_{i j}^{k} Q_{k \beta \alpha}^{\lambda}-Q_{\mu \alpha}^{\lambda} Q_{\beta i j}^{\mu}+Q_{\mu \beta}^{\lambda} Q_{\alpha i j}^{\mu}+Q_{\alpha}^{a} \partial_{a} Q_{\beta i j}^{\lambda}-Q_{\beta}^{a} \partial_{a} Q_{\alpha i j}^{\lambda}+Q_{\gamma i j}^{\lambda} Q_{\beta \alpha}^{\gamma}
\end{aligned}
$$

is (M7).

\section{Equation (V 58):}

$$
\begin{aligned}
& Q_{\beta k}^{\gamma} Q_{j \alpha}^{k}-Q_{\alpha k}^{\gamma} Q_{j \beta}^{k}+Q_{j \beta \alpha}^{\lambda} Q_{\lambda}^{\gamma}-Q_{\alpha j}^{\varepsilon} Q_{\beta \varepsilon}^{\gamma}+Q_{\beta j}^{\varepsilon} Q_{\alpha \varepsilon}^{\gamma}-Q_{j}^{a} \partial_{a} Q_{\beta \alpha}^{\gamma} \\
& \quad=-Q_{\beta \alpha}^{\varepsilon} Q_{\varepsilon j}^{\gamma}-Q_{\alpha}^{a} \partial_{a} Q_{\beta j}^{\gamma}+Q_{\beta}^{a} \partial_{a} Q_{\alpha j}^{\gamma}
\end{aligned}
$$

is (M6), providing the third term in (V 58) is changed to a negative sign.

Remark 3.9 Regarding differences in signs, it is worth observing that, given a Lie bialgebroid $\left(E, E^{*}\right)$, taking the opposite structure on either $E$ or $E^{*}$, or reversing their order, still results in a Lie bialgebroid. Thus varying the Lie bialgebroid chosen in the definition of double Lie algebroid has no important consequence, though some choices are easier to work with than others.

We now verify equations (M1) to (M7) on a basic example.

\subsection{The tangent double vector bundle of a Lie algebroid}

Let $A \rightarrow M$ be a Lie algebroid with anchor $\rho$. We have seen in Sect. 2.3.2 that

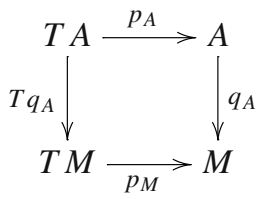

is endowed with two LA-vector bundle structures. The vertical structure $(T A \rightarrow$ $A, T M \rightarrow M$ ) is the standard tangent bundle Lie algebroid (Example 2.5) and ( $T A \rightarrow$ $T M, A \rightarrow M$ ) is the tangent prolongation of $A \rightarrow M$ as in Sect. 2.3.2. We refer to $T A$ loosely as the tangent double Lie algebroid.

Recall from Sect. 2.1.2 that a linear connection $\nabla: \mathfrak{X}(M) \times \Gamma(A) \rightarrow \Gamma(A)$ defines a decomposition $\Sigma^{\nabla}: A \times{ }_{M} T M \rightarrow T A$. This decomposition induces the two following 2-representations:

(i) The LA-vector bundle $(T A \rightarrow A, T M \rightarrow M)$ is described by the 2representation of $T M$ on $\operatorname{Id}_{A}: A \rightarrow A$ via $\left(\nabla, \nabla, R_{\nabla}\right)$ (Example 2.11 ). The anchor of $T M$ is $\operatorname{Id}_{T M}$ and the bracket is the Lie bracket of vector fields.

(ii) The LA-vector bundle $(T A \rightarrow T M, A \rightarrow T M)$ is described by the 2representation of $A$ on $\rho: A \rightarrow T M$ via $\left(\nabla^{\text {bas }}, \nabla^{\text {bas }}, R_{\nabla}^{\text {bas }}\right)$ as in Sect. 2.3.2. 
We check that these two 2-representations form a matched pair. This will provide a new proof of the fact that the tangent double of a Lie algebroid is a double Lie algebroid [16]. Conditions (M1) and (M2) in Definition 3.1 are just two times the definition of $\nabla^{\text {bas }}: \Gamma(A) \times \Gamma(A) \rightarrow \Gamma(A)$ and (M3) is the definition of $\nabla^{\text {bas }}: \Gamma(A) \times \mathfrak{X}(M) \rightarrow$ $\mathfrak{X}(M)$. Condition (M5) is the definition of $R_{\nabla}^{\text {bas }}$. Hence, we only need to check (M4), (M6) and (M7).

In the following, $X, X_{1}, X_{2}$ will be arbitrary vector fields on $M$ and $a, a_{1}, a_{2}$ arbitrary sections of $A$.

(M4) The left-hand side of (M4) is

$$
\begin{aligned}
& \nabla_{X} \nabla_{a_{1}}^{\text {bas }} a_{2}-\nabla_{a_{1}}^{\text {bas }} \nabla_{X} a_{2}-\nabla_{\nabla_{X} a_{1}}^{\text {bas }} a_{2}+\nabla_{\nabla_{a_{1}}}^{\text {bas }} a_{2} \\
& \quad=\nabla_{X}\left[a_{1}, a_{2}\right]+\nabla_{X} \nabla_{\rho\left(a_{2}\right)} a_{1}-\left[a_{1}, \nabla_{X} a_{2}\right]-\nabla_{\rho\left(\nabla_{X} a_{2}\right)} a_{1} \\
& \quad-\left[\nabla_{X} a_{1}, a_{2}\right]-\nabla_{\rho\left(a_{2}\right)} \nabla_{X} a_{1}+\nabla_{\left[\rho\left(a_{1}\right), X\right]} a_{2}+\nabla_{\rho\left(\nabla_{X} a_{1}\right)} a_{2} .
\end{aligned}
$$

The second and sixth terms add up to $R\left(X, \rho\left(a_{2}\right)\right) a_{1}+\nabla_{\left[X, \rho\left(a_{2}\right)\right]} a_{1}$ and the first, third, and fifth terms to $-R_{\nabla}^{\text {bas }}\left(a_{1}, a_{2}\right) X+\nabla_{\nabla_{a_{2}} \text { bas } X} a_{1}-\nabla_{\nabla_{a_{1}}}^{\text {bas }} a_{2}$. The definition of $\nabla^{\text {bas }}: \Gamma(A) \times \mathfrak{X}(M) \rightarrow \mathfrak{X}(M)$ yields then immediately the right hand side of (M4), namely

$$
R\left(X, \rho\left(a_{2}\right)\right) a_{1}-R_{\nabla}^{\mathrm{bas}}\left(a_{1}, a_{2}\right) X .
$$

(M6) This equation is easily verified:

$$
\begin{aligned}
- & \nabla_{a}^{\mathrm{bas}}\left[X_{1}, X_{2}\right]+\left[\nabla_{a}^{\mathrm{bas}} X_{1}, X_{2}\right]+\left[X_{1}, \nabla_{a}^{\mathrm{bas}} X_{2}\right]+\nabla_{\nabla_{X_{2}} a}^{\mathrm{bas}} X_{1}-\nabla_{\nabla_{X_{1}} a}^{\mathrm{bas}} X_{2} \\
= & -\left[\rho(a),\left[X_{1}, X_{2}\right]\right]-\rho\left(\nabla_{\left[X_{1}, X_{2}\right]} a\right)+\left[\left[\rho(a), X_{1}\right]+\rho\left(\nabla_{X_{1}} a\right), X_{2}\right] \\
& +\left[X_{1},\left[\rho(a), X_{2}\right]+\rho\left(\nabla_{X_{2}} a\right)\right]+\left[\rho\left(\nabla_{X_{2}} a\right), X_{1}\right] \\
& +\rho\left(\nabla_{X_{1}} \nabla_{X_{2}} a\right)-\left[\rho\left(\nabla_{X_{1}} a\right), X_{2}\right]-\rho\left(\nabla_{X_{2}} \nabla_{X_{1}} a\right) \\
& =\rho\left(R_{\nabla}\left(X_{1}, X_{2}\right) a\right)
\end{aligned}
$$

To get the second equality, we use the Jacobi identity for the Lie bracket of vector fields. The four remaining terms cancel pairwise.

(M7) As one would expect, checking (M7) is a long, but straightforward computation. We carry this out in detail here, but will omit similar calculations in later cases. We begin by computing

$$
\begin{aligned}
\left(\mathbf{d}_{\nabla} R_{\nabla}^{\mathrm{bas}}\right)\left(X_{1}, X_{2}\right)\left(a_{1}, a_{2}\right)= & -R_{\nabla}^{\mathrm{bas}}\left(a_{1}, a_{2}\right)\left[X_{1}, X_{2}\right] \\
& +\nabla_{X_{1}}\left(R_{\nabla}^{\mathrm{bas}}\left(a_{1}, a_{2}\right) X_{2}\right)-\nabla_{X_{2}}\left(R_{\nabla}^{\mathrm{bas}}\left(a_{1}, a_{2}\right) X_{1}\right) \\
& -R_{\nabla}^{\mathrm{bas}}\left(\nabla_{X_{1}} a_{1}, a_{2}\right) X_{2}-R_{\nabla}^{\mathrm{bas}}\left(a_{1}, \nabla_{X_{1}} a_{2}\right) X_{2} \\
& +R_{\nabla}^{\mathrm{bas}}\left(\nabla_{X_{2}} a_{1}, a_{2}\right) X_{1}+R_{\nabla}^{\text {bas }}\left(a_{1}, \nabla_{X_{2}} a_{2}\right) X_{1} .
\end{aligned}
$$


This expands out to

$$
\begin{aligned}
& \nabla_{\left[X_{1}, X_{2}\right]}\left[a_{1}, a_{2}\right]-\left[\nabla_{\left[X_{1}, X_{2}\right]} a_{1}, a_{2}\right]-\left[a_{1}, \nabla_{\left[X_{1}, X_{2}\right]} a_{2}\right]+\nabla_{\nabla_{a_{1}}^{\text {bas }}\left[X_{1}, X_{2}\right]} a_{2}-\nabla_{\nabla_{a_{2}}^{\text {bas }}\left[X_{1}, X_{2}\right]} a_{1} \\
& +\nabla_{X_{1}}\left(-\nabla_{X_{2}}\left[a_{1}, a_{2}\right]+\left[\nabla_{X_{2}} a_{1}, a_{2}\right]+\left[a_{1}, \nabla_{X_{2} a_{2}}\right]-\nabla_{\nabla_{a_{1}}^{\text {bas }} X_{2}} a_{2}+\nabla_{\nabla_{a_{2}}^{\text {bas }} X_{2}} a_{1}\right) \\
& -\nabla_{X_{2}}\left(-\nabla_{X_{1}}\left[a_{1}, a_{2}\right]+\left[\nabla_{X_{1}} a_{1}, a_{2}\right]+\left[a_{1}, \nabla_{X_{1} a_{2}}\right]-\nabla_{\nabla_{a_{1}}^{\text {bas }} X_{1}} a_{2}+\nabla_{\nabla_{a_{2}}^{\text {bas }} X_{1}} a_{1}\right) \\
& +\nabla_{X_{2}}\left[\nabla_{X_{1}} a_{1}, a_{2}\right]-\left[\nabla_{X_{2}} \nabla_{X_{1}} a_{1}, a_{2}\right]-\left[\nabla_{X_{1}} a_{1}, \nabla_{X_{2} a_{2}}\right]+\nabla_{\nabla_{\nabla_{1} a_{1}}^{\text {bas }} X_{2}} a_{2}-\nabla_{\nabla_{a_{2}}^{\text {bas }} X_{2}} \nabla_{X_{1}} a_{1} \\
& +\nabla_{X_{2}}\left[a_{1}, \nabla_{X_{1} a_{2}}\right]-\underline{\left[\nabla_{X_{2}} a_{1}, \nabla_{X_{1} a_{2}}\right]}-\left[a_{1}, \nabla_{X_{2}} \nabla_{X_{1}} a_{2}\right]+\nabla_{\nabla_{a_{1}}^{\text {bas }} X_{2}} \nabla_{X_{1}} a_{2}-\nabla_{\nabla_{\nabla_{X_{1} a_{2}}}^{\text {bas }} X_{2}} a_{1} \\
& -\nabla_{X_{1}}\left[\nabla_{X_{2}} a_{1}, a_{2}\right]+\left[\nabla_{X_{1}} \nabla_{X_{2}} a_{1}, a_{2}\right]+\left[\nabla_{X_{2}} a_{1}, \nabla_{X_{1} a_{2}}\right]-\nabla_{\nabla_{\nabla_{X} a_{1}}^{\text {bas }} X_{1}} a_{2}+\nabla_{\nabla_{a_{2}}^{\text {bas }} X_{1}} \nabla_{X_{2}} a_{1} \\
& -\underline{\nabla_{X_{1}}}\left[a_{1}, \nabla_{X_{2} a_{2}}\right]+\left[\nabla_{X_{1}} a_{1}, \nabla_{X_{2} a_{2}}\right]+\left[a_{1}, \nabla_{X_{1}} \nabla_{X_{2}} a_{2}\right]-\nabla_{\nabla_{a_{1}}^{\text {bas }} X_{1}} \nabla_{X_{2}} a_{2}+\nabla_{\nabla_{\nabla_{X_{2}} a_{2}}^{\text {bas }}} X_{1} a_{1} .
\end{aligned}
$$

Twelve terms of this equation cancel pairwise as shown, and a reordering of the remaining terms yields

$$
\begin{aligned}
- & R_{\nabla}\left(X_{1}, X_{2}\right)\left[a_{1}, a_{2}\right]+\left[R_{\nabla}\left(X_{1}, X_{2}\right) a_{1}, a_{2}\right]+\left[a_{1}, R_{\nabla}\left(X_{1}, X_{2}\right) a_{2}\right] \\
& +R_{\nabla}\left(X_{2}, \nabla_{a_{1}}^{\mathrm{bas}} X_{1}\right) a_{2}+\nabla_{\left[X_{2}, \nabla_{a_{1}}^{\mathrm{bas}} X_{1}\right]} a_{2}-R_{\nabla}\left(X_{2}, \nabla_{a_{2}}^{\mathrm{bas}} X_{1}\right) a_{1}-\nabla_{\left[X_{2}, \nabla_{a_{2}}^{\mathrm{bas}} X_{1}\right]} a_{1} \\
& -R_{\nabla}\left(X_{1}, \nabla_{a_{1}}^{\mathrm{bas}} X_{2}\right) a_{2}-\nabla_{\left[X_{1}, \nabla_{a_{1}}^{\mathrm{bas}} X_{2}\right]} a_{2}+R_{\nabla}\left(X_{1}, \nabla_{a_{2}}^{\mathrm{bas}} X_{2}\right) a_{1}+\nabla_{\left[X_{1}, \nabla_{a_{2}}\right.}^{\left.\mathrm{bas} X_{2}\right]} a_{1} \\
& +\nabla_{\nabla_{a_{1}}^{\mathrm{bas}\left[X_{1}, X_{2}\right]}} a_{2}-\nabla_{\nabla_{a_{2}}^{\mathrm{bas}}\left[X_{1}, X_{2}\right]} a_{1}-\nabla_{\nabla_{\nabla_{X_{2}} a_{1}}^{\mathrm{bas}} X_{1}} a_{2} \\
& +\nabla_{\nabla_{\nabla_{X_{2}} a_{2}}^{\mathrm{bas}} X_{1}} a_{1}+\nabla_{\nabla_{\nabla_{X_{1}} a_{1}}^{\mathrm{bas}} X_{2}} a_{2}-\nabla_{\nabla_{\nabla_{X_{1} a_{2}}}^{\mathrm{bas}} X_{2}} a_{1} .
\end{aligned}
$$

By (M6), this equals

$$
\begin{aligned}
- & R_{\nabla}\left(X_{1}, X_{2}\right)\left[a_{1}, a_{2}\right]+\left[R_{\nabla}\left(X_{1}, X_{2}\right) a_{1}, a_{2}\right]+\left[a_{1}, R_{\nabla}\left(X_{1}, X_{2}\right) a_{2}\right] \\
& +R_{\nabla}\left(X_{2}, \nabla_{a_{1}}^{\mathrm{bas}} X_{1}\right) a_{2}-R_{\nabla}\left(X_{2}, \nabla_{a_{2}}^{\mathrm{bas}} X_{1}\right) a_{1}-R_{\nabla}\left(X_{1}, \nabla_{a_{1}}^{\mathrm{bas}} X_{2}\right) a_{2} \\
& +R_{\nabla}\left(X_{1}, \nabla_{a_{2}}^{\mathrm{bas}} X_{2}\right) a_{1}+\nabla_{\rho\left(R_{\nabla}\left(X_{1}, X_{2}\right) a_{2}\right)} a_{1}-\nabla_{\rho\left(R_{\nabla}\left(X_{1}, X_{2}\right) a_{1}\right)} a_{2},
\end{aligned}
$$

which is

$$
\begin{aligned}
- & R_{\nabla}\left(X_{1}, X_{2}\right)\left[a_{1}, a_{2}\right]-\nabla_{a_{2}}^{\text {bas }} R_{\nabla}\left(X_{1}, X_{2}\right) a_{1}+\nabla_{a_{1}}^{\text {bas }} R_{\nabla}\left(X_{1}, X_{2}\right) a_{2} \\
& -R_{\nabla}\left(\nabla_{a_{1}}^{\text {bas }} X_{1}, X_{2}\right) a_{2}+R_{\nabla}\left(\nabla_{a_{2}}^{\text {bas }} X_{1}, X_{2}\right) a_{1}-R_{\nabla}\left(X_{1}, \nabla_{a_{1}}^{\text {bas }} X_{2}\right) a_{2} \\
& +R_{\nabla}\left(X_{1}, \nabla_{a_{2}}^{\text {bas }} X_{2}\right) a_{1}=\left(\mathbf{d}_{\nabla \text { bas }} R_{\nabla}\right)\left(a_{1}, a_{2}\right)\left(X_{1}, X_{2}\right) .
\end{aligned}
$$

This completes the verification that the 2-representations associated with the tangent double of a Lie algebroid form a matched pair.

Remark 3.10 Infinitesimal ideal systems [5,9] may also be understood in terms of 2-representations. It is proved in [2] that infinitesimal ideal systems are in bijective correspondence with double subbundles of $(T A ; A, T M ; M)$ of the form $\left(F ; A, F_{M} ; M\right)$, 
such that $\left(F \rightarrow A, F_{M} \rightarrow M\right)$ and $\left(F \rightarrow F_{M}, A \rightarrow M\right)$ are sub-LA-vector bundles of $(T A \rightarrow A, T M \rightarrow M)$ and $(T A \rightarrow T M, A \rightarrow M)$, respectively.

More explicitly, suppose given a decomposition of $T A$ that is adapted to the double subbundle $F$ in the sense that the LA-vector bundle $\left(F \rightarrow A, F_{M} \rightarrow M\right)$ is described by the 2-representation $\left(\operatorname{Id}_{A}: A \rightarrow A, \bar{\nabla}, \bar{\nabla}, R_{\bar{\nabla}}\right)$, where $\bar{\nabla}: \Gamma\left(F_{M}\right) \times \Gamma(A) \rightarrow \Gamma(A)$ is the restriction to $F_{M}$ of the connection $\nabla$ corresponding to the decomposition [2]. Then the LA-vector bundle $\left(F \rightarrow F_{M}, A \rightarrow T M\right)$ is described by the 2representation of $A$ on $\rho: J \rightarrow F_{M}$ via $\left(\overline{\nabla^{\text {bas }}}, \overline{\nabla^{\text {bas }}}, \overline{R_{\nabla}^{\text {bas }}}\right)$, where the $\overline{\nabla^{\text {bas }}}$ are the (well-defined!) restrictions of the basic connections to $\Gamma(A) \times \Gamma(J) \rightarrow \Gamma(J)$ and $\Gamma(A) \times \Gamma\left(F_{M}\right) \rightarrow \Gamma\left(F_{M}\right)[2]$.

It is easy to see that (M1)-(M7) for the tangent double ( $T A ; A, T M ; M)$ restrict to (M1)-(M7) for this pair of 2-representations. This refines the result in [2] to a bijective correspondence between infinitesimal ideal systems and sub-double Lie algebroids of $(T A ; A, T M ; M)$.

\subsection{The cotangent double of a Lie bialgebroid}

Let $(A, \rho,[\cdot, \cdot])$ be a Lie algebroid over a smooth manifold $M$, and assume that $A^{*}$ also has a Lie algebroid structure, with anchor denoted by $\rho_{\star}$ and with bracket $[\cdot, \cdot]_{\star}$.

Since $A^{*}$ is a Lie algebroid, $A$ has a linear Poisson structure, and so $\left(T^{*} A \rightarrow\right.$ $A, A^{*} \rightarrow M$ ) has a LA-vector bundle structure. The Lie algebroid bracket is given by

$$
\left[\mathbf{d} \ell_{\alpha_{1}}, \mathbf{d} \ell_{\alpha_{2}}\right]=\mathbf{d} \ell_{\left[\alpha_{1}, \alpha_{2}\right]}, \quad\left[\mathbf{d} \ell_{\alpha}, q_{A}^{*} \theta\right]=q_{A}^{*}\left(£_{\rho_{\star}(\alpha)} \theta\right), \quad\left[q_{A}^{*} \theta_{1}, q_{A}^{*} \theta_{2}\right]=0
$$

for all $\alpha, \alpha_{1}, \alpha_{2} \in \Gamma\left(A^{*}\right)$ and $\theta, \theta_{1}, \theta_{2} \in \Omega^{1}(M)$. The anchor is given by

$$
\Theta_{A}\left(\mathbf{d} \ell_{\alpha}\right)=\widehat{£_{\rho_{\star}(\alpha)}} \in \mathfrak{X}(A), \quad \Theta_{A}\left(q_{A}^{*} \theta\right)=\left(\rho_{\star}^{*} \theta\right)^{\uparrow} \in \mathfrak{X}(A)
$$

(see Sect. 2.1.2 for the notation). Likewise the Lie algebroid structure on $A$ induces a LA-vector bundle structure on $\left(T^{*}\left(A^{*}\right) \rightarrow A^{*}, A \rightarrow M\right)$, satisfying corresponding equations. Using the natural diffeomorphism $T^{*}\left(A^{*}\right) \rightarrow T^{*} A$ of [17], this LA-vector bundle structure may be transferred to $\left(T^{*} A \rightarrow A^{*}, A \rightarrow M\right)$ and equips the double vector bundle

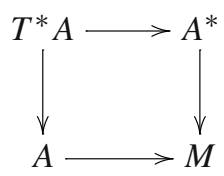

with two LA-vector bundle structures. If $\left(A, A^{*}\right)$ is a Lie bialgebroid then (18) is a double Lie algebroid with core $T^{*} M$ ([16]; see also [23]). We now establish an equivalent result in terms of 2-representations.

A linear connection $\nabla: \mathfrak{X}(M) \times \Gamma(A) \rightarrow \Gamma(A)$, is equivalent to a decomposition $\Sigma_{\nabla}$ of $T A$ and so to a decomposition $\Sigma_{\nabla}^{\star}$ of (18). Using (8), one can check that $\Sigma_{\nabla}^{\star}: A \times A^{*} \rightarrow T^{*} A$ sends $\left(a_{m}, \alpha_{m}\right)$ to $\mathbf{d}_{a_{m}} \ell_{\alpha}-\left(T_{a_{m}} q\right)^{*}\left\langle\nabla . \alpha, a_{m}\right\rangle$ for any $\alpha \in \Gamma\left(A^{*}\right)$ such that $\alpha(m)=\alpha_{m}$ (see also [6]). 
A computation shows that the representation up to homotopy defined by $\left(T^{*} A \rightarrow\right.$ $\left.A, A^{*} \rightarrow M\right)$ and the decomposition $\Sigma^{\star}$ is given by the morphism $\rho_{\star}^{*}: T^{*} M \rightarrow A$, by the connections

$$
\left(\nabla^{*}\right)^{\text {bas* }}: \Gamma\left(A^{*}\right) \times \Gamma(A) \rightarrow \Gamma(A), \quad\left(\nabla^{*}\right)^{\text {bas* }}: \Gamma\left(A^{*}\right) \times \Omega^{1}(M) \rightarrow \Omega^{1}(M),
$$

and by

$$
R_{\nabla^{*}}^{\text {bas* }} \in \Omega^{2}\left(A^{*}, \operatorname{Hom}\left(A, T^{*} M\right)\right) .
$$

(Recall from the previous example that since we have an ordinary connection $\nabla^{*}$ - the dual connection to $\nabla-$ on $A^{*}$, we can define the basic $A^{*}$-connections on $A^{*}$ and $T M$. The two connections above are their duals.)

Next the LA-vector bundle $(T A \rightarrow T M, A \rightarrow M)$ dualises over $A$ to $\left((T A) \gtrless_{A} \rightarrow\right.$ $\left.A^{*}, A \rightarrow M\right)$, which is $\left(T^{*} A \rightarrow A^{*}, A \rightarrow M\right)$. Recall that the decomposition $\Sigma^{\nabla}$ of $T A$ and this LA-vector bundle structure define the 2-representation ( $\left.\rho: A \rightarrow T M, \nabla^{\text {bas }}, \nabla^{\text {bas }}, R_{\nabla}^{\text {bas }}\right)$. The discussion in Sect. 2.3.1 yields then that the 2representation of $A$ describing $\left(T^{*} A \rightarrow A^{*}, A \rightarrow M\right)$ in terms of the decomposition $\Sigma_{\nabla}^{\star}$ is on the complex $\rho^{*}: T^{*} M \rightarrow A^{*}$ and is defined by the connections

$$
\nabla^{\text {bas* }}: \Gamma(A) \times \Gamma\left(A^{*}\right) \rightarrow \Gamma\left(A^{*}\right), \quad \nabla^{\text {bas* }}: \Gamma(A) \times \Omega^{1}(M) \rightarrow \Omega^{1}(M),
$$

and the curvature term

$$
-R_{\nabla}^{\text {bas* }} \in \Omega^{2}\left(A, \operatorname{Hom}\left(A^{*}, T^{*} M\right)\right) .
$$

A long, but straightforward computation shows that (M1)-(M7) for the two 2-representations in (19)-(20) and in (21)-(22) are equivalent to (B1)-(B3) below, and hence to $\left(A, A^{*}\right)$ being a Lie bialgebroid. Hence we recover the fact that $\left(T^{*} A ; A^{*}, A ; M\right)$ is a double Lie algebroid if and only if $\left(A, A^{*}\right)$ is a Lie bialgebroid. Conversely, (M1)-(M7) provide interesting identities relating the basic curvatures and connections defined by two dual connections on a Lie bialgebroid. As a summary, we have the following corollary of our main theorem.

Theorem 3.11 Let $(A, \rho,[\cdots])$ and $\left(A, \rho_{\star},[\cdots]_{\star}\right)$ be two Lie algebroids in duality. Then $\left(A, A^{*}\right)$ is a Lie bialgebroid if and only iffor any connection $\nabla: \mathfrak{X}(M) \times \Gamma(A) \rightarrow$ $\Gamma(A)$, the 2-representation $\left(\rho_{\star}^{*}: T^{*} M \rightarrow A,\left(\nabla^{*}\right)^{\text {bas* }},\left(\nabla^{*}\right)^{\text {bas* }}, R_{\nabla^{*}}^{\text {bas }}\right)$ of $A^{*}$ and the 2-representation $\left(\rho^{*}: T^{*} M \rightarrow A, \nabla^{\text {bas* }}, \nabla^{\text {bas* }},-R_{\nabla}^{\text {bas* }}\right.$ ) of A form a matched pair.

\section{Proof of the main theorem}

We will prove the theorem by checking the Lie bialgebroid condition only on particular families of sections; the linear sections and the core sections. The main difficulty is to understand the additional conditions which have to be verified by the families of sections for the proof to be complete. This is done in Sect. 4.1. In Sect. 4.2, we will show how the equations found in Sect. 4.1 imply (M1)-(M7) and vice-versa. 


\subsection{Families of sections of Lie bialgebroids}

We recall the definition of a Lie bialgebroid [17]; see also [15, Chapter 12]. We will then show how the equation defining a Lie bialgebroid $\left(A, A^{*}\right)$ need be verified only on families of spanning sections of $A$ and $A^{*}$.

Definition 4.1 Let $q_{A}: A \rightarrow M$ and $q_{A^{*}}: A^{*} \rightarrow M$ be a pair of dual vector bundles, and suppose each has a Lie algebroid structure, with anchors $\rho: A \rightarrow T M$ and $\rho_{*}: A^{*} \rightarrow T M$ respectively, and brackets $[\cdot, \cdot]$ and $[\cdot, \cdot]_{*}$.

Then $\left(A, A^{*}\right)$ is a Lie bialgebroid if for all $a_{1}, a_{2} \in \Gamma(A)$ :

$$
\mathbf{d}_{A^{*}}\left[a_{1}, a_{2}\right]=\left[\mathbf{d}_{A^{*}} a_{1}, a_{2}\right]+\left[a_{1}, \mathbf{d}_{A^{*}} a_{2}\right] .
$$

The brackets on the RHS are extensions to 2-vectors by standard Schouten calculus.

It is often very convenient to check this condition only on the elements of a given set of sections $\mathcal{S} \subseteq \Gamma(A)$ which spans $\Gamma(A)$ as a $C^{\infty}(M)$-module. We will formalize this technique shortly. We first need to recall some consequences of the definition.

The proof of the following proposition is a straightforward computation.

Proposition 4.2 Let $A$ and $A^{*}$ be dual vector bundles with Lie algebroid structures. For $a_{1}, a_{2} \in \Gamma(A), \alpha_{1}, \alpha_{2} \in \Gamma\left(A^{*}\right)$ and $f \in C^{\infty}(M)$, we have

$$
\begin{aligned}
\left(\mathbf{d}_{A^{*}}\right. & {\left.\left[a_{1}, f a_{2}\right]-\left[\mathbf{d}_{A^{*}} a_{1}, f a_{2}\right]-\left[a_{1}, \mathbf{d}_{A^{*}}\left(f a_{2}\right)\right]\right)\left(\alpha_{1}, \alpha_{2}\right) } \\
= & \left.f \cdot\left(\mathbf{d}_{A^{*}}\left[a_{1}, a_{2}\right]-\left[\mathbf{d}_{A^{*}} a_{1}, a_{2}\right]-\left[a_{1}, \mathbf{d}_{A^{*}} a_{2}\right]\right)\right)\left(\alpha_{1}, \alpha_{2}\right) \\
& -\left\langle a_{2}, \alpha_{2}\right\rangle \cdot\left(\left[\rho\left(a_{1}\right), \rho_{*}\left(\alpha_{1}\right)\right](f)-\rho_{*}\left(\mathfrak{f}_{a_{1}} \alpha_{1}\right)(f)+\rho\left(\mathfrak{f}_{\alpha_{1}} a_{1}\right)(f)-\rho_{*}\left(\mathbf{d}_{A} f\right)\left\langle a_{1}, \alpha_{1}\right\rangle\right) \\
& +\left\langle a_{2}, \alpha_{1}\right\rangle \cdot\left(\left[\rho\left(a_{1}\right), \rho_{*}\left(\alpha_{2}\right)\right](f)-\rho_{*}\left(\mathfrak{f}_{a_{1}} \alpha_{2}\right)(f)+\rho\left(\mathfrak{f}_{\alpha_{2}} a_{1}\right)(f)-\rho_{*}\left(\mathbf{d}_{A} f\right)\left\langle a_{1}, \alpha_{2}\right\rangle\right) .
\end{aligned}
$$

Now assume that $\left(A, A^{*}\right)$ is a Lie bialgebroid. Take any $a_{1} \in \Gamma A$ and any nonvanishing local section $\alpha_{1} \in \Gamma\left(A^{*}\right)$. Choose a (local) nonvanishing $a_{2} \in \Gamma(A)$ and an $\alpha_{2} \in \Gamma\left(A^{*}\right)$ such that $\left\langle a_{2}, \alpha_{1}\right\rangle=0$ and $\left\langle a_{2}, \alpha_{2}\right\rangle=1$. (If $A$ has rank 1 then (25) below is vacuously true.) Equation (24) now reduces to

$$
\left[\rho\left(a_{1}\right), \rho_{*}\left(\alpha_{1}\right)\right](f)-\rho_{*}\left(\mathfrak{f}_{a_{1}} \alpha_{1}\right)(f)+\rho\left(\mathfrak{f}_{\alpha_{1}} a_{1}\right)(f)-\rho_{*}\left(\mathbf{d}_{A} f\right)\left\langle a_{1}, \alpha_{1}\right\rangle=0
$$

for all $a_{1} \in \Gamma(A), f \in C^{\infty}(M)$, and local nonvanishing $\alpha_{1} \in \Gamma\left(A^{*}\right)$. A straightworward computation shows that the left-hand side of (25) is tensorial in the term $\alpha_{1}$. Hence, (25) holds for all $\alpha_{1} \in \Gamma\left(A^{*}\right)$. (For another proof, see [15, 12.1.8].)

On the other hand, the left-hand side of (25) is not tensorial in the term $a_{1}$. We multiply $a_{1}$ by a function $g \in C^{\infty}(M)$ in this equation, expand out, and subtract

$$
g \cdot\left(\left[\rho\left(a_{1}\right), \rho_{*}\left(\alpha_{1}\right)\right](f)-\rho_{*}\left(£_{a_{1}} \alpha_{1}\right)(f)+\rho\left(£_{\alpha_{1}} a_{1}(f)-\rho_{*}\left(\mathbf{d}_{A} f\right)\left\langle a_{1}, \alpha_{1}\right\rangle\right)=0 .\right.
$$

We get that

$$
\left\langle a_{1}, \alpha_{1}\right\rangle \cdot\left(-\rho_{*}\left(\mathbf{d}_{A} g\right)(f)-\rho_{*}\left(\mathbf{d}_{A} f\right)(g)\right)=0 .
$$


Again, since $a_{1}$ and $\alpha_{1}$ were arbitrary, we have found

$$
-\rho_{*}\left(\mathbf{d}_{A} g\right)(f)=\rho_{*}\left(\mathbf{d}_{A} f\right)(g) \text { for all } f, g \in C^{\infty}(M),
$$

which is easily seen to be equivalent to

$$
-\rho \circ \rho_{*}^{*}=\rho_{*} \circ \rho^{*}
$$

see also [17], $[15, \S 12.1]$. The map $\rho_{*} \circ \rho^{*}: T^{*} M \rightarrow T M$ defines a Poisson structure on $M$, which we take to be the Poisson structure on $M$ induced by the Lie bialgebroid structure.

These considerations lead to the following result.

Proposition 4.3 Let $q_{A}: A \rightarrow M$ and $q_{A^{*}}: A^{*} \rightarrow M$ be a pair of dual vector bundles, and suppose each has a Lie algebroid structure, with anchors $\rho: A \rightarrow T M$ and $\rho_{*}: A^{*} \rightarrow T M$ respectively, and brackets $[\cdot, \cdot]$ and $[\cdot, \cdot]_{*}$. Let $\mathcal{S}$ be a subset of $\Gamma(A)$ which spans $\Gamma(A)$ as a $C^{\infty}(M)$-module.

Then $\left(A, A^{*}\right)$ is a Lie bialgebroid if and only if the following three conditions hold.

(BI) $\mathbf{d}_{A^{*}}\left[a_{1}, a_{2}\right]=\left[\mathbf{d}_{A^{*}} a_{1}, a_{2}\right]+\left[a_{1}, \mathbf{d}_{A^{*}} a_{2}\right]$ for all $a_{1}, a_{2} \in \mathcal{S}$,

(B2) $\left[\rho(a), \rho_{*}(\alpha)\right](f)-\rho_{*}\left(£_{a} \alpha\right)(f)+\rho\left(£_{\alpha} a\right)(f)-\rho_{*}\left(\mathbf{d}_{A} f\right)\langle a, \alpha\rangle=0$ for all $a \in \mathcal{S}, \alpha \in \Gamma\left(A^{*}\right)$ and $f \in C^{\infty}(M)$, and

(B3) $-\rho \circ \rho_{*}^{*}=\rho_{*} \circ \rho^{*}$.

Proof We proved above that these three conditions hold when $\left(A, A^{*}\right)$ is a Lie bialgebroid. For the converse, a quick computation using (B1) and the considerations before the proposition shows that

$$
\begin{aligned}
& \left(\mathbf{d}_{A^{*}}\left[g a_{1}, f a_{2}\right]-\left[\mathbf{d}_{A^{*}}\left(g a_{1}\right), f a_{2}\right]-\left[g a_{1}, \mathbf{d}_{A^{*}}\left(f a_{2}\right)\right]\right)\left(\alpha_{1}, \alpha_{2}\right) \\
& \left.=f g \cdot\left(\mathbf{d}_{A^{*}}\left[a_{1}, a_{2}\right]-\left[\mathbf{d}_{A^{*}} a_{1}, a_{2}\right]-\left[a_{1}, \mathbf{d}_{A^{*}} a_{2}\right]\right)\right)\left(\alpha_{1}, \alpha_{2}\right) \\
& -f g\left\langle a_{2}, \alpha_{2}\right\rangle \cdot\left(\left[\rho\left(a_{1}\right), \rho_{*}\left(\alpha_{1}\right)\right](f)-\rho_{*}\left(\mathfrak{f}_{a_{1}} \alpha_{1}\right)(f)+\rho\left(\mathfrak{f}_{\alpha_{1}} a_{1}\right)(f)-\rho_{*}\left(\mathbf{d}_{A} f\right)\left\langle a_{1}, \alpha_{1}\right\rangle\right) \\
& +f g\left\langle a_{2}, \alpha_{1}\right\rangle \cdot\left(\left[\rho\left(a_{1}\right), \rho_{*}\left(\alpha_{2}\right)\right](f)-\rho_{*}\left(\mathfrak{f}_{a_{1}} \alpha_{2}\right)(f)+\rho\left(\mathfrak{f}_{\alpha_{2}} a_{1}\right)(f)-\rho_{*}\left(\mathbf{d}_{A} f\right)\left\langle a_{1}, \alpha_{2}\right\rangle\right) \\
& +f g\left\langle a_{1}, \alpha_{1}\right\rangle \cdot\left(\left[\rho\left(a_{2}\right), \rho_{*}\left(\alpha_{2}\right)\right](f)-\rho_{*}\left(\mathfrak{f}_{a_{2}} \alpha_{2}\right)(f)+\rho\left(\mathfrak{f}_{\alpha_{2}} a_{2}\right)(f)-\rho_{*}\left(\mathbf{d}_{A} f\right)\left\langle a_{2}, \alpha_{2}\right\rangle\right) \\
& -f g\left\langle a_{1}, \alpha_{2}\right\rangle \cdot\left(\left[\rho\left(a_{2}\right), \rho_{*}\left(\alpha_{1}\right)\right](f)-\rho_{*}\left(\mathfrak{f}_{a_{2}} \alpha_{1}\right)(f)+\rho\left(\mathfrak{f}_{\alpha_{1}} a_{2}\right)(f)-\rho_{*}\left(\mathbf{d}_{A} f\right)\left\langle a_{2}, \alpha_{1}\right\rangle\right) \text {. }
\end{aligned}
$$

for all $a_{1}, a_{2} \in \mathcal{S}, \alpha_{1}, \alpha_{2} \in \Gamma\left(A^{*}\right)$ and $f, g \in C^{\infty}(M)$. This vanishes by (B1) and (B2). Since the Lie bialgebroid condition is additive and $\Gamma(A)$ is spanned as a $C^{\infty}(M)$-module by $\mathcal{S}$, we are done.

Remark 4.4 In Proposition 4.3, the first two conditions are $C^{\infty}(M)$-linear in the $\Gamma\left(A^{*}\right)$-argument, so it is sufficient to check them on a subset $\mathcal{R} \subseteq \Gamma\left(A^{*}\right)$ that spans $\Gamma\left(A^{*}\right)$ as a $C^{\infty}(M)$-module. 


\subsection{The Lie bialgebroid conditions on lifts and on core sections}

We write here $\Theta_{A}: D * A \rightarrow T C^{*}$ for the anchor of $D * A \rightarrow C^{*}$ and $\Theta_{B}: D * B \rightarrow$ $T C^{*}$ for the anchor of $D * B \rightarrow C^{*}$. We set

$$
\mathcal{S}:=\Gamma_{C^{*}}^{c}\left(D^{*} A\right) \cup \sigma_{A}^{\star}(\Gamma(A))
$$

and

$$
\mathcal{R}:=\Gamma_{C^{*}}^{c}(D * B) \cup \sigma_{B}^{\star}(\Gamma(B)) .
$$

Proposition 4.5 Condition (B3) on $\mathcal{S}$ and $\mathcal{R}$ is equivalent to $\rho_{A} \circ \partial_{A}=\rho_{B} \circ \partial_{B}$ and (M1).

Proof Since $\Theta_{A} \circ \Theta_{B}^{*}$ and $\Theta_{B} \circ \Theta_{A}^{*}$ are vector bundle maps $T^{*} C^{*} \rightarrow T C^{*}$, it is sufficient to check (B3) on $\mathbf{d} F$ for $F \in C^{\infty}\left(C^{*}\right)$. In fact, it is even sufficient to check (B3) on $\mathbf{d}\left(q_{C^{*}}^{*} f\right)$ for $f \in C^{\infty}(M)$ and $\mathbf{d} \ell_{c}$ for $c \in \Gamma(C)$.

Choose first $f \in C^{\infty}(M)$ and consider $q_{C^{*}}^{*} f \in C^{\infty}\left(C^{*}\right)$. We have for any section $b \in \Gamma(B)$ :

$$
\left\langle\Theta_{B}^{*}\left(\mathbf{d} q_{C^{*}}^{*} f\right), \sigma_{B}^{\star}(b)\right\rangle=\widehat{\nabla_{b}^{*}}\left(q_{C^{*}}^{*} f\right)=q_{C^{*}}^{*}\left(\rho_{B}(b) f\right)
$$

and for any $\alpha \in \Gamma\left(A^{*}\right)$ :

$$
\left\langle\Theta_{B}^{*}\left(\mathbf{d} q_{C^{*}}^{*} f\right), \alpha^{\dagger}\right\rangle=\left(\partial_{A}^{*} \alpha\right)^{\uparrow}\left(q_{C^{*}}^{*} f\right)=0 .
$$

This shows that

$$
\Theta_{B}^{*}\left(\mathbf{d} q_{C^{*}}^{*} f\right)=\left(\rho_{B}^{*} \mathbf{d} f\right)^{\dagger} \in \Gamma_{C^{*}}^{c}(D * A) .
$$

We get consequently $\Theta_{A} \circ \Theta_{B}^{*}\left(\mathbf{d} q_{C^{*}}^{*} f\right)=\left(\partial_{B}^{*} \rho_{B}^{*} \mathbf{d} f\right)^{\uparrow} \in \mathfrak{X}\left(C^{*}\right)$. In the same manner, we find $\Theta_{A} \circ \Theta_{B}^{*}\left(\mathbf{d} q_{C^{*}}^{*} f\right)=\left(-\partial_{A}^{*} \rho_{A}^{*} \mathbf{d} f\right)^{\uparrow} \in \mathfrak{X}\left(C^{*}\right)$. The equality of $\Theta_{A} \circ \Theta_{B}^{*}$ and $-\Theta_{B} \circ \Theta_{A}^{*}$ on pullbacks is hence equivalent to $\rho_{A} \circ \partial_{A}=\rho_{B} \circ \partial_{B}$.

We continue with linear functions. Choose $c \in \Gamma(C)$. Then for any section $b \in$ $\Gamma(B)$, we get

$$
\left\langle\Theta_{B}^{*}\left(\mathbf{d} \ell_{c}\right), \sigma_{B}^{\star}(b)\right\rangle=\widehat{\nabla_{b}^{*}}\left(\ell_{c}\right)=\ell_{\nabla_{b} c}
$$

and for any $\alpha \in \Gamma\left(A^{*}\right)$ :

$$
\left\langle\Theta_{B}^{*}\left(\mathbf{d} \ell_{c}\right), \alpha^{\dagger}\right\rangle=\left(\partial_{A}^{*} \alpha\right)^{\uparrow}\left(\ell_{c}\right)=q_{C}^{*}\left\langle\alpha, \partial_{A} c\right\rangle .
$$

This shows

$$
\Theta_{B}^{*}\left(\mathbf{d} \ell_{c}\right)=-\sigma_{A}^{\star}\left(\partial_{A} c\right)+\widetilde{\langle\nabla \cdot c, \cdot\rangle} \in \Gamma_{C^{*}}^{l}(D * A),
$$

where $\langle\nabla . c, \cdot\rangle$ is seen as an element of $\Gamma\left(\operatorname{Hom}\left(C^{*}, B^{*}\right)\right)$. This leads to

$$
\Theta_{A} \circ \Theta_{B}^{*}\left(\mathbf{d} \ell_{c}\right)\left(\ell_{c^{\prime}}\right)=-\ell_{\nabla_{\partial_{A}(c)} c^{\prime}}+\ell_{\nabla_{\partial_{B}\left(c^{\prime}\right)}}
$$


for all $c^{\prime} \in \Gamma(C)$ and

$$
\Theta_{A} \circ \Theta_{B}^{*}\left(\mathbf{d} \ell_{c}\right)\left(q_{C^{*}}^{*} f\right)=-q_{C^{*}}^{*}\left(\rho_{A} \circ \partial_{A}(c) f\right)
$$

for $f \in C^{\infty}(M)$. We find similar equations for $\Theta_{B} \circ \Theta_{A}^{*}\left(\mathbf{d} \ell_{c}\right)\left(\ell_{c^{\prime}}\right)$ and $\Theta_{B} \circ$ $\Theta_{A}^{*}\left(\mathbf{d} \ell_{C}\right)\left(q_{C^{*}}^{*} f\right)$, and can conclude that $\Theta_{A} \circ \Theta_{B}^{*}=-\Theta_{B} \circ \Theta_{A}^{*}$ holds if and only if $\rho_{A} \circ \partial_{A}=\rho_{B} \circ \partial_{B}$ and (M1) are satisfied.

As a corollary of this proof, we find the following result. Recall that the map $\Theta_{B} \circ$ $\Theta_{A}^{*}: T^{*} C^{*} \rightarrow T C$ defines a Poisson structure on $C^{*}$ (see (26) and the considerations following it).

Corollary 4.6 The Poisson structure on $C^{*}$ induced by the Lie bialgebroid structure is the linear Poisson structure dual to the Lie algebroid structure on C as in Remark 3.5. More explicitly, it is given by

$$
\begin{aligned}
\left\{\ell_{c_{1}}, \ell_{c_{2}}\right\} & =\left(\Theta_{B} \circ \Theta_{A}^{*}\right)\left(q_{C^{*}}^{*} \mathbf{d} \ell_{c_{1}}\right)\left(\ell_{c_{2}}\right)=\ell_{\nabla_{\partial_{A}\left(c_{1}\right)}\left(c_{2}\right)-\nabla_{\partial_{B}\left(c_{2}\right)}\left(c_{1}\right)=\ell_{\left[c_{1}, c_{2}\right]},}, \\
\left\{\ell_{c_{1}}, q_{C^{*}}^{*} f\right\} & =\left(\Theta_{B} \circ \Theta_{A}^{*}\right)\left(q_{C^{*}}^{*} \mathbf{d} \ell_{c_{1}}\right)\left(q_{C^{*}}^{*} f\right)=q_{C^{*}}^{*}\left(\rho_{A}\left(\partial_{A}(c)\right)(f)\right) \\
\left\{q_{C^{*}}^{*} f_{1}, q_{C^{*}}^{*} f_{2}\right\} & =\left(\Theta_{B} \circ \Theta_{A}^{*}\right)\left(q_{C^{*}}^{*} \mathbf{d} f_{1}\right)\left(q_{C^{*}}^{*} f\right)=0 .
\end{aligned}
$$

Remark 4.7 Note that the apparent asymmetry between the structures over $A$ and $B$ arises from unavoidable choices in the identifications between the various duals. The Poisson structure on $C^{*}$ is nonetheless determined by requiring $\partial_{A}$ and $\partial_{B}$ to be morphisms of Lie algebroids.

For the study of (B1) and (B2), we will need the following lemma. Recall that for a Lie algebroid $A$, the Lie derivative $£: \Gamma(A) \times \Gamma\left(A^{*}\right) \rightarrow \Gamma\left(A^{*}\right)$ is defined by

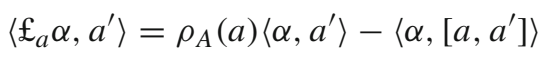

for all $a, a^{\prime} \in \Gamma(A)$ and $\alpha \in \Gamma\left(A^{*}\right)$.

Lemma 4.8 The Lie derivative $£: \Gamma_{C^{*}}(D * A) \times \Gamma_{C^{*}}(D * B) \rightarrow \Gamma_{C^{*}}\left(D *^{*} B\right)$ is given by the following identities:

$$
\begin{aligned}
£_{\beta^{\dagger}} \alpha^{\dagger} & =0, \quad £_{\beta^{\dagger}} \sigma_{B}^{\star}(b)=-\left\langle b, \nabla_{.}^{*} \beta\right\rangle^{\dagger}, \quad £_{\sigma_{A}^{\star}(a)} \alpha^{\dagger}=£_{a} \alpha^{\dagger}, \\
£_{\sigma_{A}^{\star}(a)} \sigma_{B}^{\star}(b) & =\sigma_{B}^{\star}\left(\nabla_{a} b\right)+\widetilde{R(a, \cdot) b}
\end{aligned}
$$

for all $a \in \Gamma(A), b \in \Gamma(B), \alpha \in \Gamma\left(A^{*}\right)$ and $\beta \in \Gamma\left(B^{*}\right)$. The Lie derivative $£: \Gamma_{C^{*}}$ $(D * B) \times \Gamma_{C^{*}}\left(D^{*} A\right) \rightarrow \Gamma_{C^{*}}(D * A)$ is given by:

$$
\begin{aligned}
£_{\alpha^{\dagger}} \beta^{\dagger} & =0, \quad £_{\alpha^{\dagger}} \sigma_{A}^{\star}(a)=-\left\langle a, \nabla_{.}^{*} \alpha\right\rangle^{\dagger}, \quad £_{\sigma_{A}^{\star}(b)} \beta^{\dagger}=£_{b} \beta^{\dagger} \\
£_{\sigma_{A}^{\star}(b)} \sigma_{A}^{\star}(a) & =\sigma_{B}^{\star}\left(\nabla_{b} a\right)+\widehat{R(b, \cdot) a} .
\end{aligned}
$$

Note that in these equations, $R(a, \cdot) b$ is seen as a section of $\operatorname{Hom}\left(C^{*}, A^{*}\right)$ and $R(b, \cdot) a$ is seen as a section of $\operatorname{Hom}\left(C^{*}, B^{*}\right)$. 
Proof We have

$$
\begin{aligned}
\left\langle\beta_{2}^{\dagger}, £_{\beta_{1}^{\dagger}} \alpha^{\dagger}\right\rangle & =\left(\partial_{B}^{*} \beta_{1}\right)^{\uparrow}\left\langle\beta_{2}^{\dagger}, \alpha^{\dagger}\right\rangle-\left\langle\left[\beta_{1}^{\dagger}, \beta_{2}^{\dagger}\right], \alpha^{\dagger}\right\rangle=0 \quad \text { and } \\
\left\langle\sigma_{A}^{\star}(a), £_{\beta_{1}^{\dagger}} \alpha^{\dagger}\right\rangle & =\left(\partial_{B}^{*} \beta_{1}\right)^{\uparrow}\left(-q_{C^{*}}^{*}\langle\alpha, a\rangle\right)+\left\langle\nabla_{a}^{*} \beta_{1}^{\dagger}, \alpha^{\dagger}\right\rangle=0
\end{aligned}
$$

for arbitrary $\beta_{1}, \beta_{2} \in \Gamma\left(B^{*}\right), \alpha \in \Gamma\left(A^{*}\right)$ and $a \in \Gamma(A)$. This proves $£_{\beta_{1}^{\dagger}} \alpha^{\dagger}=0$.

Then we compute

$$
\left\langle\beta_{2}^{\dagger}, £_{\beta_{1}^{\dagger}} \sigma_{B}^{\star}(b)\right\rangle=\left(\partial_{B}^{*} \beta_{1}\right)^{\dagger}\left(q_{C^{*}}^{*}\left\langle\beta_{2}, b\right\rangle\right)-\left\langle\left[\beta_{1}^{\dagger}, \beta_{2}^{\dagger}\right], \sigma_{B}^{\star}(b)\right\rangle=0
$$

which shows that $£_{\beta_{1}^{\dagger}} \sigma_{B}^{\star}(b)$ is a section with values in the core, and

$$
\left\langle\sigma_{A}^{\star}(a), £_{\beta_{1}^{\dagger}} \sigma_{B}^{\star}(b)\right\rangle=0+\left\langle\nabla_{a}^{*} \beta_{1}^{\dagger}, \sigma_{B}^{\star}(b)\right\rangle=q_{C^{*}}^{*}\left\langle b, \nabla_{a}^{*} \beta_{1}\right\rangle .
$$

This proves $£_{\beta_{1}^{\dagger}} \sigma_{B}^{\star}(b)=-\left\langle b, \nabla^{*} \beta_{1}\right\rangle^{\dagger}$, with $\left\langle b, \nabla^{*} \beta_{1}\right\rangle \in \Gamma\left(A^{*}\right)$. We also find

$$
\begin{aligned}
& \left\langle\beta^{\dagger}, £_{\sigma_{A}^{\star}\left(a_{1}\right)} \alpha^{\dagger}\right\rangle=\widehat{\nabla_{a_{1}}^{*}}\left\langle\beta^{\dagger}, \alpha^{\dagger}\right\rangle-\left\langle\nabla_{a_{1}}^{*} \beta^{\dagger}, \alpha^{\dagger}\right\rangle=0 \quad \text { and } \\
& \left\langle\sigma_{A}^{\star}\left(a_{2}\right), £_{\sigma_{A}^{\star}\left(a_{1}\right)} \alpha^{\dagger}\right\rangle=-\widehat{\nabla_{a_{1}}^{*}}\left(q_{C^{*}}^{*}\left\langle\alpha, a_{2}\right\rangle\right)-\left\langle\sigma_{A}^{\star}\left[a_{1}, a_{2}\right]+\left(R\left(a_{1}, a_{2}\right)^{*}\right)^{\dagger}, \alpha^{\dagger}\right\rangle \\
& =-q_{C^{*}}^{*}\left(\rho_{A}\left(a_{1}\right)\left\langle\alpha, a_{2}\right\rangle-\left\langle\alpha,\left[a_{1}, a_{2}\right]\right\rangle\right)=-q_{C^{*}}^{*}\left\langle £_{a_{1}} \alpha, a_{2}\right\rangle
\end{aligned}
$$

for arbitrary $a_{1}, a_{2} \in \Gamma(A)$ and $\alpha \in \Gamma\left(A^{*}\right)$. This proves the equality $£_{\sigma_{A}^{\star}\left(a_{1}\right)} \alpha^{\dagger}=$ $£_{a_{1}} \alpha^{\dagger}$.

The identity

$$
\begin{aligned}
\left\langle\beta^{\dagger}, £_{\sigma_{A}^{\star}\left(a_{1}\right)} \sigma_{B}^{\star}(b)\right\rangle & =\widehat{\nabla_{a_{1}}^{*}} q_{C^{*}}^{*}\langle\beta, b\rangle-\left\langle\nabla_{a_{1}}^{*} \beta^{\dagger}, \sigma_{B}^{\star}(b)\right\rangle \\
& =q_{C^{*}}^{*}\left(\rho_{A}\left(a_{1}\right)\langle\beta, b\rangle-\left\langle\nabla_{a_{1}}^{*} \beta, b\right\rangle\right)=q_{C^{*}}^{*}\left\langle\beta, \nabla_{a_{1}} b\right\rangle
\end{aligned}
$$

shows that $£_{\sigma_{A}^{\star}\left(a_{1}\right)} \sigma_{B}^{\star}(b)$ is the sum of $\sigma_{B}^{\star}\left(\nabla_{a_{1}} b\right)$ with a section with values in the core. To find out this core term, we finally compute

$$
\begin{aligned}
\left\langle\sigma_{A}^{\star}\left(a_{2}\right), £_{\sigma_{A}^{\star}\left(a_{1}\right)} \sigma_{B}^{\star}(b)\right\rangle & =0-\left\langle\sigma_{A}^{*}\left[a_{1}, a_{2}\right]+R \widetilde{R\left(a_{1}, a_{2}\right)^{*}}, \sigma_{B}^{\star}(b)\right\rangle \\
& =-\ell_{R\left(a_{1}, a_{2}\right)(b)} .
\end{aligned}
$$

This shows that $£_{\sigma_{A}^{\star}\left(a_{1}\right)} \sigma_{B}^{\star}(b)=\sigma_{B}^{\star}\left(\nabla_{a_{1}} b\right)+\widetilde{R\left(a_{1}, \cdot\right)} b$.

The formulas describing the Lie derivative $£: \Gamma_{C^{*}}(D * B) \times \Gamma_{C^{*}}(D * A) \rightarrow$ $\Gamma_{C^{*}}(D * A)$ can be verified in the same manner.

Proposition 4.9 Condition (B2) on $\mathcal{S}$ and $\mathcal{R}$ is equivalent to (M2), (M3), (M4) and (M0).

Proof The idea of this proof is to check (B2) on linear and core sections in $\mathcal{S}$ and $\mathcal{R}$,

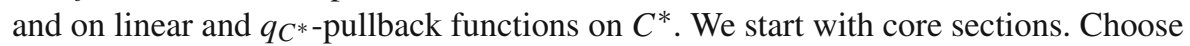
$\alpha \in \Gamma\left(A^{*}\right)$ and $\beta \in \Gamma\left(B^{*}\right)$. We have $\left[\Theta_{B}\left(\alpha^{\dagger}\right), \Theta_{A}\left(\beta^{\dagger}\right)\right]=\left[\left(\partial_{A}^{*} \alpha\right)^{\uparrow},\left(\partial_{B}^{*} \beta\right)^{\uparrow}\right]=0$. 
By Lemma 4.8 and with $\left\langle\beta^{\dagger}, \alpha^{\dagger}\right\rangle=0$, we find that (B2) is trivially satisfied on $\alpha^{\dagger}, \beta^{\dagger}$ and any element of $C^{\infty}\left(C^{*}\right)$.

Now choose $a \in \Gamma(A), \alpha \in \Gamma\left(A^{*}\right)$. Using Lemma 4.8 we find for all $F \in C^{\infty}\left(C^{*}\right)$

$$
\begin{aligned}
{\left[\Theta_{B}\left(\alpha^{\dagger}\right), \Theta_{A}\left(\sigma_{A}^{\star}(a)\right)\right](F)-\Theta_{A}\left(£_{\alpha^{\dagger}} \sigma_{A}^{\star}(a)\right)(F)+\Theta_{B}\left(£_{\sigma_{A}^{\star}(a)} \alpha^{\dagger}\right)(F) } \\
\quad-\Theta_{A}\left(\mathbf{d}_{D}{\mho_{B}}_{B} F\right)\left\langle\sigma_{A}^{\star}(a), \alpha^{\dagger}\right\rangle \\
\quad=-\left(\nabla_{a}^{*}\left(\partial_{A}^{*} \alpha\right)\right)^{\uparrow}(F)+\left\langle a, \nabla_{\partial_{B}}^{*} \alpha\right\rangle^{\uparrow}(F)+\left(\partial_{A}^{*} £_{a} \alpha\right)^{\uparrow}(F)+\left(\Theta_{A} \circ \Theta_{B}^{*} \mathbf{d} F\right) q_{C^{*}}^{*}\langle\alpha, a\rangle .
\end{aligned}
$$

In particular, for $F=q_{C^{*}}^{*} f, f \in C^{\infty}(M)$, this is $0+\left(\partial_{B}^{*} \rho_{B}^{*} \mathbf{d} f\right)^{\uparrow} q_{C^{*}}^{*}\langle\alpha, a\rangle=0$ by (27) and for $F=\ell_{c}, c \in \Gamma(C)$, this is

$$
\left.q_{C^{*}}^{*}\left(-\left\langle\nabla_{a}^{*}\left(\partial_{A}^{*} \alpha\right)\right), c\right\rangle+\left\langle a, \nabla_{\partial_{B}}^{*} \alpha\right\rangle+\left\langle\partial_{A}^{*} £_{a} \alpha, c\right\rangle-\left(\rho_{B} \circ \partial_{B}(c)\right)\langle\alpha, a\rangle\right)
$$

by (28). But this equals $q_{C^{*}}^{*}\left(\left\langle\alpha, \partial_{A}\left(\nabla_{a} c\right)-\nabla_{\partial_{B} c} a-\left[a, \partial_{A} c\right]\right\rangle\right)$. This shows that (B2) is in this case equivalent to (M2). In the same manner, (B2) on $\beta^{\dagger} \in \mathcal{S}$ for $\beta \in \Gamma\left(B^{*}\right)$, $\sigma_{B}^{\star}(b) \in \mathcal{R}$ for $b \in \Gamma(B)$ and $F \in C^{\infty}\left(C^{*}\right)$ is equivalent to (M3).

Now choose $a \in \Gamma(A)$ and $b \in \Gamma(B)$. (B2) on $\sigma_{A}^{\star}(a), \sigma_{B}^{\star}(b)$ and $q_{C^{*}}^{*} f, f \in$ $C^{\infty}(M)$, is

$$
q_{C^{*}}^{*}\left(\left(\left[\rho_{B}(b), \rho_{A}(a)\right]-\rho_{A}\left(\nabla_{b} a\right)+\rho_{B}\left(\nabla_{a} b\right)\right)(f)\right)=0
$$

by Lemma 4.8. This is (M0). Finally we compute (B2) on $\sigma_{A}^{\star}(a), \sigma_{B}^{\star}(b)$ and $\ell_{c}$, for $c \in \Gamma(C)$. This is

$$
\ell_{\nabla_{b} \nabla_{a} c-\nabla_{a} \nabla_{b} c}-\ell_{\nabla_{\nabla_{b} a} c+R_{B A}\left(b, \partial_{B} c\right) a}+\ell_{\nabla_{\nabla_{a} b} c+R_{A B}\left(a, \partial_{A} c\right) b}=0
$$

by Lemma 4.8. We find hence that (B2) on $\sigma_{A}^{\star}(a), \sigma_{B}^{\star}(b)$ and $\ell_{c}$ is equivalent to (M4).

We conclude the proof of Theorem 3.6 with the study of (B1) on linear and core sections.

Proposition 4.10 Condition (B1) on elements of $\mathcal{S}$ and $\mathcal{R}$ is equivalent to (M5), (M6) and (M7).

In the proof of this proposition, we will use the following formulas. Let $A$ and $A^{*}$ be a pair of Lie algebroids in duality. Then, for all $a \in \Gamma(A)$ and $\alpha_{1}, \alpha_{2} \in \Gamma\left(A^{*}\right)$ :

$$
\left(\mathbf{d}_{A^{*}} a\right)\left(\alpha_{1}, \alpha_{2}\right)=\rho_{A^{*}}\left(\alpha_{1}\right)\left\langle\alpha_{2}, a\right\rangle-\rho_{A^{*}}\left(\alpha_{2}\right)\left\langle\alpha_{1}, a\right\rangle-\left\langle\left[\alpha_{1}, \alpha_{2}\right]_{A^{*}}, a\right\rangle .
$$

For all $a_{1}, a_{2} \in \Gamma(A)$ and $\alpha_{1}, \alpha_{2} \in \Gamma\left(A^{*}\right)$, we have

$$
\begin{aligned}
& {\left[\mathbf{d}_{A^{*}} a_{1}, a_{2}\right]_{A}\left(\alpha_{1}, \alpha_{2}\right)=-\left(\mathfrak{f}_{a_{2}} \mathbf{d}_{A^{*}} a_{1}\right)\left(\alpha_{1}, \alpha_{2}\right)} \\
& =-£_{\rho_{A}\left(a_{2}\right)}\left(\mathbf{d}_{A^{*} a_{1}}\left(\alpha_{1}, \alpha_{2}\right)\right)+\mathbf{d}_{A^{*} a_{1}}\left(£_{a_{2}} \alpha_{1}, \alpha_{2}\right) \\
& +\mathbf{d}_{A^{*}} a_{1}\left(\alpha_{1}, £_{a_{2}} \alpha_{2}\right) \text {. }
\end{aligned}
$$


Proof First choose $\alpha_{1}, \alpha_{2} \in \Gamma\left(A^{*}\right)$. We have $\mathbf{d}_{D} *_{A}\left[\alpha_{1}^{\dagger}, \alpha_{2}^{\dagger}\right]=0$. For $\beta_{1}, \beta_{2} \in \Gamma\left(B^{*}\right)$ and $a_{1}, a_{2} \in \Gamma(A)$, we find using Lemma 4.8

$$
\begin{aligned}
& {\left[\mathbf{d}_{D} *_{A} \alpha_{1}^{\dagger}, \alpha_{2}^{\dagger}\right]\left(\beta_{1}^{\dagger}, \beta_{2}^{\dagger}\right)=-\left(\partial_{A}^{*} \alpha_{2}\right)^{\uparrow}\left(\mathbf{d}_{D} *_{A} \alpha_{1}^{\dagger}\left(\beta_{1}^{\dagger}, \beta_{2}^{\dagger}\right)\right)+\mathbf{d}_{D} *_{A} \alpha_{1}^{\dagger}\left(£_{\alpha_{2}^{\dagger}} \beta_{1}^{\dagger}, \beta_{2}^{\dagger}\right)} \\
& +\mathbf{d}_{D} \nVdash_{A} \alpha_{1}^{\dagger}\left(\beta_{1}^{\dagger}, £_{\alpha_{2}^{\dagger}} \beta_{2}^{\dagger}\right)=0 \\
& {\left[\mathbf{d}_{D} *_{A} \alpha_{1}^{\dagger}, \alpha_{2}^{\dagger}\right]\left(\sigma_{A}^{\star}\left(a_{1}\right), \beta_{2}^{\dagger}\right)=-\left(\partial_{A}^{*} \alpha_{2}\right)^{\dagger}\left(\mathbf{d}_{D} *_{A} \alpha_{1}^{\dagger}\left(\sigma_{A}^{\star}\left(a_{1}\right), \beta_{2}^{\dagger}\right)\right)} \\
& +\mathbf{d}_{D{ }_{A}} \alpha_{1}^{\dagger}\left(£_{\alpha_{2}^{\dagger}} \sigma_{A}^{\star}\left(a_{1}\right), \beta_{2}^{\dagger}\right) \\
& +\mathbf{d}_{D} *_{A} \alpha_{1}^{\dagger}\left(\sigma_{A}^{\star}\left(a_{1}\right), £_{\alpha_{2}^{\dagger}} \beta_{2}^{\dagger}\right)=0 \text {, }
\end{aligned}
$$

and

$$
\begin{aligned}
& {\left[\mathbf{d}_{D} \mho_{A} \alpha_{1}^{\dagger}, \alpha_{2}^{\dagger}\right]\left(\sigma_{A}^{\star}\left(a_{1}\right), \sigma_{A}^{\star}\left(a_{2}\right)\right)=-\left(\partial_{A}^{*} \alpha_{2}\right)^{\uparrow}\left(\mathbf{d}_{D} \mho_{A} \alpha_{1}^{\dagger}\left(\sigma_{A}^{\star}\left(a_{1}\right), \sigma_{A}^{\star}\left(a_{2}\right)\right)\right)} \\
& +\mathbf{d}_{D} \ngtr_{A} \alpha_{1}^{\dagger}\left(£_{\alpha_{2}^{\dagger}} \sigma_{A}^{\star}\left(a_{1}\right), \sigma_{A}^{\star}\left(a_{2}\right)\right) \\
& +\mathbf{d}_{D} *_{A} \alpha_{1}^{\dagger}\left(\sigma_{A}^{\star}\left(a_{1}\right), £_{\alpha_{2}^{\dagger}} \sigma_{A}^{\star}\left(a_{2}\right)\right) \\
& =-\left(\partial_{A}^{*} \alpha_{2}\right)^{\uparrow} q_{C^{*}}^{*}\left(-\rho_{A}\left(a_{1}\right)\left\langle\alpha_{1}, a_{2}\right\rangle\right. \\
& \left.+\rho_{A}\left(a_{2}\right)\left\langle\alpha_{1}, a_{1}\right\rangle+\left\langle\alpha_{1},\left[a_{1}, a_{2}\right]\right\rangle\right) \\
& +\mathbf{d}_{D} *_{A} \alpha_{1}^{\dagger}\left(-\left\langle a_{1}, \nabla_{.}^{*} \alpha_{2}\right\rangle^{\dagger}, \sigma_{A}^{\star}\left(a_{2}\right)\right) \\
& +\mathbf{d}_{D} *_{A} \alpha_{1}^{\dagger}\left(\sigma_{A}^{\star}\left(a_{1}\right),-\left\langle a_{2}, \nabla_{.}^{*} \alpha_{2}\right\rangle^{\dagger}\right)=0 \text {. }
\end{aligned}
$$

Thus, we have $\mathbf{d}_{D} \mho_{A}\left[\alpha_{1}^{\dagger}, \alpha_{2}^{\dagger}\right]=0=\left[\mathbf{d}_{D}{\mho_{A}}_{A} \alpha_{1}^{\dagger}, \alpha_{2}^{\dagger}\right]+\left[\alpha_{1}^{\dagger}, \mathbf{d}_{D} \mho_{A} \alpha_{2}^{\dagger}\right]$. Choose now $\alpha \in \Gamma\left(A^{*}\right)$ and $b \in \Gamma(B)$. We have $\mathbf{d}_{D} *_{A}\left[\sigma_{B}^{\star}(b), \alpha^{\dagger}\right]=\mathbf{d}_{D} *_{A}\left(\nabla_{b}^{*} \alpha\right)^{\dagger}$, and so in particular $\mathbf{d}_{D}{\mho_{A}}_{A}\left[\sigma_{B}^{\star}(b), \alpha^{\dagger}\right]\left(\beta_{1}^{\dagger}, \beta_{2}^{\dagger}\right)=0, \mathbf{d}_{D} \mho_{A}\left[\sigma_{B}^{\star}(b), \alpha^{\dagger}\right]\left(\sigma_{A}^{\star}\left(a_{1}\right), \beta_{2}^{\dagger}\right)=0$ and

$$
\begin{aligned}
\mathbf{d}_{D} *_{A}\left[\sigma_{B}^{\star}(b), \alpha^{\dagger}\right]\left(\sigma_{A}^{\star}\left(a_{1}\right), \sigma_{A}^{\star}\left(a_{2}\right)\right)= & q_{C^{*}}^{*}\left(-\rho_{A}\left(a_{1}\right)\left\langle\nabla_{b}^{*} \alpha, a_{2}\right\rangle\right. \\
& \left.+\rho_{A}\left(a_{2}\right)\left\langle\nabla_{b}^{*} \alpha, a_{1}\right\rangle+\left\langle\nabla_{b}^{*} \alpha,\left[a_{1}, a_{2}\right]\right\rangle\right) \\
= & -q_{C^{*}}^{*}\left(\mathbf{d}_{A}\left(\nabla_{b}^{*} \alpha\right)\left(a_{1}, a_{2}\right)\right) .
\end{aligned}
$$

On the other hand, we can check as above that $\left[\mathbf{d}_{D}{\mho_{A}}_{A_{B}} \sigma_{B}^{\star}(b), \alpha^{\dagger}\right]\left(\beta_{1}^{\dagger}, \beta_{2}^{\dagger}\right)=0$, that $\left[\mathbf{d}_{D} \nVdash_{A} \sigma_{B}^{\star}(b), \alpha^{\dagger}\right]\left(\sigma_{A}^{\star}\left(a_{1}\right), \beta_{2}^{\dagger}\right)=0$ and that

$$
\begin{aligned}
& {\left[\mathbf{d}_{D}{{ }_{A}}_{A} \sigma_{B}^{\star}(b), \alpha^{\dagger}\right]\left(\sigma_{A}^{\star}\left(a_{1}\right), \sigma_{A}^{\star}\left(a_{2}\right)\right)} \\
& \quad=-\left(\partial_{A}^{*} \alpha\right)^{\uparrow}\left(\mathbf{d}_{D} \nVdash_{A} \sigma_{B}^{\star}(b)\left(\sigma_{A}^{\star}\left(a_{1}\right), \sigma_{A}^{\star}\left(a_{2}\right)\right)\right) \\
& \quad+\mathbf{d}_{D} \nVdash_{A} \sigma_{B}^{\star}(b)\left(£_{\alpha^{\dagger}} \sigma_{A}^{\star}\left(a_{1}\right), \sigma_{A}^{\star}\left(a_{2}\right)\right)+\mathbf{d}_{D} \nVdash_{A} \sigma_{B}^{\star}(b)\left(\sigma_{A}^{\star}\left(a_{1}\right), £_{\alpha^{\dagger}} \sigma_{A}^{\star}\left(a_{2}\right)\right)
\end{aligned}
$$




$$
\begin{aligned}
= & \left(\partial_{A}^{*} \alpha\right)^{\dagger}\left\langle\left[\sigma_{A}^{\star}\left(a_{1}\right), \sigma_{A}^{\star}\left(a_{2}\right)\right], \sigma_{B}^{\star}(b)\right\rangle \\
& +\mathbf{d}_{D} *_{A} \sigma_{B}^{\star}(b)\left(-\left\langle a_{1}, \nabla_{.}^{*} \alpha\right\rangle^{\dagger}, \sigma_{A}^{\star}\left(a_{2}\right)\right)+\mathbf{d}_{D} *_{A} \sigma_{B}^{\star}(b)\left(\sigma_{A}^{\star}\left(a_{1}\right),-\left\langle a_{2}, \nabla_{.}^{*} \alpha\right\rangle^{\dagger}\right) \\
= & q_{C^{*}}^{*}\left(\left\langle\partial_{A}^{*} \alpha, R\left(a_{1}, a_{2}\right) b\right\rangle+\rho_{A}\left(a_{2}\right)\left\langle a_{1}, \nabla_{b}^{*} \alpha\right\rangle-\left\langle b, \nabla_{a_{2}}^{*}\left\langle a_{1}, \nabla_{.}^{*} \alpha\right\rangle\right\rangle\right. \\
& \left.-\rho_{A}\left(a_{1}\right)\left\langle a_{2}, \nabla_{b}^{*} \alpha\right\rangle+\left\langle b, \nabla_{a_{1}}^{*}\left\langle a_{2}, \nabla_{.}^{*} \alpha\right\rangle\right\rangle\right) \\
= & q_{C^{*}}^{*}\left(\left\langle\partial_{A}^{*} \alpha, R\left(a_{1}, a_{2}\right) b\right\rangle+\left\langle a_{1}, \nabla_{\nabla_{a_{2}} b}^{*} \alpha\right\rangle-\left\langle a_{2}, \nabla_{\nabla_{a_{1}} b}^{*} \alpha\right\rangle\right) .
\end{aligned}
$$

Now using (10) and (11) we finally get $\left[\sigma_{B}^{\star}(b), \mathbf{d}_{D} *_{A} \alpha^{\dagger}\right]\left(\beta_{1}^{\dagger}, \beta_{2}^{\dagger}\right)=0$,

$$
\begin{aligned}
{\left[\sigma_{B}^{\star}(b), \mathbf{d}_{D} \mho_{A} \alpha^{\dagger}\right]\left(\sigma_{A}^{\star}\left(a_{1}\right), \beta_{2}^{\dagger}\right)=} & \widehat{\nabla}_{b}^{*}\left(\mathbf{d}_{D} \mho_{A} \alpha^{\dagger}\left(\sigma_{A}^{\star}\left(a_{1}\right), \beta_{2}^{\dagger}\right)\right) \\
& -\mathbf{d}_{D} \mho_{A} \alpha^{\dagger}\left(\sigma_{A}^{\star}\left(\nabla_{b} a_{1}\right)+\widetilde{R(b, \cdot) a_{1}}, \beta_{2}^{\dagger}\right) \\
& -\mathbf{d}_{D} \nVdash_{A} \alpha^{\dagger}\left(\sigma_{A}^{\star}\left(a_{1}\right), £_{b} \beta_{2}^{\dagger}\right)=0
\end{aligned}
$$

and

$$
\begin{aligned}
& {\left[\sigma_{B}^{\star}(b), \mathbf{d}_{D} *_{A} \alpha^{\dagger}\right]\left(\sigma_{A}^{\star}\left(a_{1}\right), \sigma_{A}^{\star}\left(a_{2}\right)\right)} \\
& =\widehat{\nabla}_{b}^{*}\left(\mathbf{d}_{D} *_{A} \alpha^{\dagger}\left(\sigma_{A}^{\star}\left(a_{1}\right), \sigma_{A}^{\star}\left(a_{2}\right)\right)\right)-\mathbf{d}_{D} \mho_{A} \alpha^{\dagger}\left(\sigma_{A}^{\star}\left(\nabla_{b} a_{1}\right)+\widetilde{R(b, \cdot) a_{1}}, \sigma_{A}^{\star}\left(a_{2}\right)\right) \\
& \quad-\mathbf{d}_{D} *_{A} \alpha^{\dagger}\left(\sigma_{A}^{\star}\left(a_{1}\right), \sigma_{A}^{\star}\left(\nabla_{b} a_{2}\right)+\widehat{R(b, \cdot) a_{2}}\right) \\
& =q_{C^{*}}^{*}\left(-\rho_{B}(b)\left(\mathbf{d}_{A} \alpha\left(a_{1}, a_{2}\right)\right)+\rho_{A}\left(\nabla_{b} a_{1}\right)\left\langle\alpha, a_{2}\right\rangle-\rho_{A}\left(a_{2}\right)\left\langle\nabla_{b} a_{1}, \alpha\right\rangle\right. \\
& \left.\quad-\left\langle\alpha,\left[\nabla_{b} a_{1}, a_{2}\right]\right\rangle+\rho_{A}\left(a_{1}\right)\left\langle\nabla_{b} a_{2}, \alpha\right\rangle-\rho_{A}\left(\nabla_{b} a_{2}\right)\left\langle a_{1}, \alpha\right\rangle-\left\langle\alpha,\left[a_{1}, \nabla_{b} a_{2}\right]\right\rangle\right) .
\end{aligned}
$$

We hence find that

$$
\mathbf{d}_{D} *_{A}\left[\sigma_{B}^{\star}(b), \alpha^{\dagger}\right]=\left[\mathbf{d}_{D} *_{A} \sigma_{B}^{\star}(b), \alpha^{\dagger}\right]+\left[\sigma_{B}^{\star}(b), \mathbf{d}_{D} *_{A} \alpha^{\dagger}\right]
$$

if and only if

$$
\begin{aligned}
& \mathbf{d}_{A}\left(\nabla_{b}^{*} \alpha\right)\left(a_{1}, a_{2}\right)+\left\langle\partial_{A}^{*} \alpha, R\left(a_{1}, a_{2}\right) b\right\rangle+\left\langle a_{1}, \nabla_{\nabla_{a_{2}} b}{ }^{*} \alpha\right\rangle-\left\langle a_{2}, \nabla_{\nabla_{a_{1}}}^{*} \alpha\right\rangle \\
& \quad-\rho_{B}(b)\left(\mathbf{d}_{A} \alpha\left(a_{1}, a_{2}\right)\right)+\rho_{A}\left(\nabla_{b} a_{1}\right)\left\langle\alpha, a_{2}\right\rangle-\rho_{A}\left(a_{2}\right)\left\langle\nabla_{b} a_{1}, \alpha\right\rangle-\left\langle\alpha,\left[\nabla_{b} a_{1}, a_{2}\right]\right\rangle \\
& \quad+\rho_{A}\left(a_{1}\right)\left\langle\nabla_{b} a_{2}, \alpha\right\rangle-\rho_{A}\left(\nabla_{b} a_{2}\right)\left\langle a_{1}, \alpha\right\rangle-\left\langle\alpha,\left[a_{1}, \nabla_{b} a_{2}\right]\right\rangle=0
\end{aligned}
$$

for all $a_{1}, a_{2} \in \Gamma\left(A^{*}\right)$. This is

$$
\begin{aligned}
& \left\langle\alpha, \partial_{A} R\left(a_{1}, a_{2}\right) b+\nabla_{b}\left[a_{1}, a_{2}\right]-\left[\nabla_{b} a_{1}, a_{2}\right]-\left[a_{1}, \nabla_{b} a_{2}\right]+\nabla_{\nabla_{1} b} a_{2}-\nabla_{\nabla_{a_{2}} b} a_{1}\right\rangle \\
& \quad+\left(\left[\rho_{A}\left(a_{1}\right), \rho_{B}(b)\right]-\rho_{B}\left(\nabla_{a_{1}} b\right)+\rho_{A}\left(\nabla_{b} a_{1}\right)\right)\left\langle\alpha, a_{2}\right\rangle \\
& \quad-\left(\left[\rho_{A}\left(a_{2}\right), \rho_{B}(b)\right]-\rho_{B}\left(\nabla_{a_{2}} b\right)+\rho_{A}\left(\nabla_{b} a_{2}\right)\right)\left\langle\alpha, a_{1}\right\rangle=0 .
\end{aligned}
$$


By replacing in this equation $a_{2}$ by $f a_{2}$ with $f \in C^{\infty}(M)$, we find (M0) since $a_{1}, a_{2}, b$ and $\alpha$ were arbitrary. Then, using (M0) twice, we obtain (M5).

We conclude the proof of the theorem with the most technical formula. Choose $b_{1}, b_{2} \in \Gamma(B)$. We want to study the equation

$$
\mathbf{d}_{D} \nVdash_{A}\left[\sigma_{B}^{\star}\left(b_{1}\right), \sigma_{B}^{\star}\left(b_{2}\right)\right]=\left[\mathbf{d}_{D} *_{A} \sigma_{B}^{\star}\left(b_{1}\right), \sigma_{B}^{\star}\left(b_{2}\right)\right]+\left[\sigma_{B}^{\star}\left(b_{1}\right), \mathbf{d}_{D} \nVdash_{A} \sigma_{B}^{\star}\left(b_{2}\right)\right] .
$$

We have $\mathbf{d}_{D} *_{A}\left[\sigma_{B}^{\star}\left(b_{1}\right), \sigma_{B}^{\star}\left(b_{2}\right)\right]=\mathbf{d}_{D} *_{A}\left(\sigma_{B}^{\star}\left[b_{1}, b_{2}\right]+R\left(b_{1}, b_{2}\right)^{* \dagger}\right)$ and we find easily that both sides of (30) vanish on $\beta_{1}^{\dagger}, \beta_{2}^{\dagger}$, for $\beta_{1}, \beta_{2} \in \Gamma\left(B^{*}\right)$. We have for $a \in \Gamma(A)$ and $\beta \in \Gamma\left(B^{*}\right)$ :

$$
\begin{aligned}
\mathbf{d}_{D} *_{A} & \left.\left(\sigma_{B}^{\star}\left[b_{1}, b_{2}\right]+R_{B A} \widetilde{\left(b_{1}, b_{2}\right.}\right)^{*}\right)\left(\sigma_{A}^{\star}(a), \beta^{\dagger}\right) \\
& =q_{C^{*}}^{*}\left(\rho_{A}(a)\left\langle\left[b_{1}, b_{2}\right], \beta\right\rangle+\left\langle\partial_{B}^{*} \beta, R\left(b_{1}, b_{2}\right) a\right\rangle-\left\langle\left[b_{1}, b_{2}\right], \nabla_{a}^{*} \beta\right\rangle\right) \\
& =q_{C^{*}}^{*}\left(\left\langle\nabla_{a}\left[b_{1}, b_{2}\right], \beta\right\rangle+\left\langle\partial_{B}^{*} \beta, R\left(b_{1}, b_{2}\right) a\right\rangle\right)
\end{aligned}
$$

and

$$
\begin{aligned}
& {\left[\mathbf{d}_{D} \mho_{A} \sigma_{B}^{\star}\left(b_{1}\right), \sigma_{B}^{\star}\left(b_{2}\right)\right]\left(\sigma_{A}^{\star}(a), \beta^{\dagger}\right)=-\widehat{\nabla_{b_{2}}^{*}}\left(\mathbf{d}_{D} \nVdash_{A} \sigma_{B}^{\star}\left(b_{1}\right)\left(\sigma_{A}^{\star}(a), \beta^{\dagger}\right)\right)} \\
& +\mathbf{d}_{D} \nVdash_{A} \sigma_{B}^{\star}\left(b_{1}\right)\left(\sigma_{A}^{\star}\left(\nabla_{b_{2}} a\right)+\widetilde{R\left(b_{2}, \cdot\right)} a, \beta^{\dagger}\right) \\
& +\mathbf{d}_{D} \nVdash_{A} \sigma_{B}^{\star}\left(b_{1}\right)\left(\sigma_{A}^{\star}(a), £_{b_{2}} \beta^{\dagger}\right) \text {. }
\end{aligned}
$$

Now, using (11) this becomes,

$$
\begin{aligned}
q_{C^{*}}^{*}( & -\rho_{B}\left(b_{2}\right) \rho_{A}(a)\left\langle b_{1}, \beta\right\rangle+\rho_{B}\left(b_{2}\right)\left\langle b_{1}, \nabla_{a}^{*} \beta\right\rangle+\rho_{A}\left(\nabla_{b_{2}} a\right)\left\langle b_{1}, \beta\right\rangle-\underline{\left\langle\partial_{B}^{*} \beta, R\left(b_{2}, b_{1}\right) a\right\rangle} \\
- & \left.\left\langle b_{1}, \nabla_{\nabla_{b_{2}} a}^{*} \beta\right\rangle+\left\langle\partial_{B}^{*} \beta, R\left(b_{2}, b_{1}\right) a\right\rangle+\rho_{A}(a)\left\langle b_{1}, £_{b_{2}} \beta\right\rangle-\left\langle b_{1}, \nabla_{a}^{*} £_{b_{2}} \beta\right\rangle\right) \\
& =q_{C^{*}}^{*}\left(-\rho_{B}\left(b_{2}\right)\left\langle\nabla_{a} b_{1}, \beta\right\rangle+\left\langle\nabla_{\nabla_{b_{2}} a} b_{1}, \beta\right\rangle+\left\langle\nabla_{a} b_{1}, £_{b_{2}} \beta\right\rangle\right) \\
& =q_{C^{*}}^{*}\left(-\left\langle\left[b_{2}, \nabla_{a} b_{1}\right], \beta\right\rangle+\left\langle\nabla_{\nabla_{b_{2}} a} b_{1}, \beta\right\rangle\right) .
\end{aligned}
$$

Thus, we find that the two sides of (30) are equal on $\left(\sigma_{A}^{\star}(a), \beta^{\dagger}\right)$ if and only if (M6) is satisfied.

Finally we consider $a_{1}, a_{2} \in \Gamma(A)$. We have

$$
\begin{aligned}
& \left.\mathbf{d}_{D} *_{A}\left(\sigma_{B}^{\star}\left[b_{1}, b_{2}\right]+R \widehat{\left(b_{1}, b_{2}\right.}\right)^{*}\right)\left(\sigma_{A}^{\star}\left(a_{1}\right), \sigma_{A}^{\star}\left(a_{2}\right)\right) \\
& \quad=-\widehat{\nabla_{a_{1}}^{*}} \ell_{R\left(b_{1}, b_{2}\right) a_{2}}+\widehat{\nabla_{a_{2}}^{*}} \ell_{R\left(b_{1}, b_{2}\right) a_{1}}+\ell_{R\left(b_{1}, b_{2}\right)\left[a_{1}, a_{2}\right]-R\left(a_{1}, a_{2}\right)\left[b_{1}, b_{2}\right]}=\ell_{c}
\end{aligned}
$$

where $c=-\nabla_{a_{1}}\left(R\left(b_{1}, b_{2}\right) a_{2}\right)+\nabla_{a_{2}}\left(R\left(b_{1}, b_{2}\right) a_{1}\right)+R\left(b_{1}, b_{2}\right)\left[a_{1}, a_{2}\right]-R\left(a_{1}, a_{2}\right)$ $\left[b_{1}, b_{2}\right] \in \Gamma(C)$. On the other hand, 


$$
\begin{aligned}
& {\left[\mathbf{d}_{D} \mho_{A} \sigma_{B}^{\star}\left(b_{1}\right), \sigma_{B}^{\star}\left(b_{2}\right)\right]\left(\sigma_{A}^{\star}\left(a_{1}\right), \sigma_{A}^{\star}\left(a_{2}\right)\right)=-\widehat{\nabla_{b_{2}}^{*}}\left(\mathbf{d}_{D} \mho_{A} \sigma_{B}^{\star}\left(b_{1}\right)\left(\sigma_{A}^{\star}\left(a_{1}\right), \sigma_{A}^{\star}\left(a_{2}\right)\right)\right)} \\
& +\mathbf{d}_{D} \nVdash_{A} \sigma_{B}^{\star}\left(b_{1}\right)\left(\sigma_{A}^{\star}\left(\nabla_{b_{2}} a_{1}\right)+R \widetilde{R\left(b_{2}, \cdot\right)} a_{1}, \sigma_{A}^{\star}\left(a_{2}\right)\right) \\
& +\mathbf{d}_{D} \nVdash_{A} \sigma_{B}^{\star}\left(b_{1}\right)\left(\sigma_{A}^{\star}\left(a_{1}\right), \sigma_{A}^{\star}\left(\nabla_{b_{2}} a_{2}\right)+\widetilde{R\left(b_{2}, \cdot\right)} a_{2}\right)
\end{aligned}
$$

and by (10) this is

$$
\begin{aligned}
& \left.\left.\widehat{\nabla_{b_{2}}^{*}} \ell_{R_{A B}\left(a_{1}, a_{2}\right) b_{1}}-\widehat{\nabla_{a_{2}}^{*}} \ell_{R\left(b_{2}, b_{1}\right) a_{1}}-\left\langle R\left(\widehat{\left(\nabla_{b_{2}} a_{1}, a_{2}\right.}\right)^{*}-\nabla_{a_{2}}^{\operatorname{Hom}} \widehat{\left(R \left(b_{2}\right.\right.}, \cdot\right) a_{1}\right), \sigma_{B}^{\star}\left(b_{1}\right)\right\rangle \\
& \left.\left.+\widehat{\nabla_{a_{1}}^{*}} \ell_{R\left(b_{2}, b_{1}\right) a_{2}}+\left\langle R\left(\widehat{\nabla_{b_{2}} a_{2}, a_{1}}\right)^{*}-\nabla_{a_{1}}^{\operatorname{Hom}} \widetilde{\left(R \left(b_{2}\right.\right.}, \cdot\right) a_{2}\right), \sigma_{B}^{\star}\left(b_{1}\right)\right\rangle \\
& =\ell_{c_{1}+c_{2}} \text {, }
\end{aligned}
$$

where

$$
\begin{aligned}
c_{1} & =\nabla_{b_{2}}\left(R\left(a_{1}, a_{2}\right) b_{1}\right)-\nabla_{a_{2}}\left(R\left(b_{2}, b_{1}\right) a_{1}\right)-R\left(\nabla_{b_{2}} a_{1}, a_{2}\right) b_{1}+\left\langle b_{1}, \nabla_{a_{2}}^{\mathrm{Hom}}\left(R\left(b_{2}, \cdot\right) a_{1}\right)\right\rangle \\
& =\nabla_{b_{2}}\left(R\left(a_{1}, a_{2}\right) b_{1}\right)+R\left(\nabla_{a_{2}} b_{1}, b_{2}\right) a_{1}-R\left(\nabla_{b_{2}} a_{1}, a_{2}\right) b_{1}, \\
c_{2} & =\nabla_{a_{1}}\left(R\left(b_{2}, b_{1}\right) a_{2}\right)+R\left(\nabla_{b_{2}} a_{2}, a_{1}\right) b_{1}-\left\langle b_{1}, \nabla_{a_{1}}^{\mathrm{Hom}} R\left(b_{2}, \cdot\right) a_{2}\right\rangle \\
& =R\left(\nabla_{a_{1}} b_{1}, b_{2}\right) a_{2}-R\left(a_{1}, \nabla_{b_{2}} a_{2}\right) b_{1} .
\end{aligned}
$$

Hence, we find that the two sides of (30) coincide on $\left(\sigma_{A}^{\star}\left(a_{1}\right), \sigma_{A}^{\star}\left(a_{2}\right)\right)$ if and only if (M7) is satisfied.

\section{This completes the proof of Theorem 3.6.}

Acknowledgements The Research of M. JL, was partially supported by a fellowship for prospective researchers (PBELP2_137534) of the Swiss NSF for a postdoctoral stay at UC Berkeley, and by a DorotheaSchlözer fellowship of the University of Göttingen. The authors are very grateful to Th. Voronov, and to an anonymous referee, for helpful comments.

Open Access This article is distributed under the terms of the Creative Commons Attribution 4.0 International License (http://creativecommons.org/licenses/by/4.0/), which permits unrestricted use, distribution, and reproduction in any medium, provided you give appropriate credit to the original author(s) and the source, provide a link to the Creative Commons license, and indicate if changes were made.

\section{References}

1. Arias, A.C., Crainic, M.: Representations up to Homotopy of Lie Algebroids. J. Reine Angew. Math. 663, 91-126 (2012)

2. Drummond, T., Jotz, M., Ortiz, C.: VB-Algebroid Morphisms and Representations up to Homotopy. Differ. Geom. Appl. 40, 332-357 (2015)

3. Evens, S., Lu, J.-H., Weinstein, A.: Transverse Measures, the Modular Class and a Cohomology Pairing for Lie Algebroids. Q. J. Math. Oxf. Ser. 50(200), 417-436 (1999)

4. Gracia-Saz, A., Mehta, R.A.: Lie Algebroid Structures on Double Vector Bundles and Representation Theory of Lie Algebroids. Adv. Math. 223(4), 1236-1275 (2010)

5. Hawkins, E.: A Groupoid Approach to Quantization. J. Symplectic Geom. 6(1), 61-125 (2008)

6. Jotz, M.: Dorfman Connections and Courant Algebroids (2013). arXiv:1209.6077

7. Jotz Lean, M.: N-manifolds of Degree 2 and Metric Double Vector Bundles (2015). arXiv:1504.00880

8. Jotz Lean, M., Mackenzie, K.C.H.: Transitive Double Lie Algebroids (2014) (in preparation) 
9. Jotz Lean, M., Ortiz, C.: Foliated Groupoids and Infinitesimal Ideal Systems. Indag. Math. (N. S.) 25(5), 1019-1053 (2014)

10. Lu, J.-H.: Poisson Homogeneous Spaces and Lie Algebroids Associated to Poisson Actions. Duke Math. J. 86(2), 261-304 (1997)

11. Mackenzie, K.C.H.: Double Lie Algebroids and Second-Order Geometry. I. Adv. Math. 94(2), 180-239 (1992)

12. Mackenzie, K.C.H.: Double Lie Algebroids and Iterated Tangent Bundles (1998). arXiv:math/9808081

13. Mackenzie, K.C.H.: On symplectic Double Groupoids and the Duality of Poisson Groupoids. Int. J. Math. 10(4), 435-456 (1999)

14. Mackenzie, K.C.H.: Double Lie Algebroids and Second-Order Geometry. II. Adv. Math. 154(1), 46-75 (2000)

15. Mackenzie, K.C.H.: General Theory of Lie Groupoids and Lie Algebroids, vol 231 of London Mathematical Society Lecture Note Series. Cambridge University Press, Cambridge (2005)

16. Mackenzie, K.C.H.: Ehresmann Doubles and Drinfel'd Doubles for Lie Algebroids and Lie Bialgebroids. J. Reine Angew. Math. 658, 193-245 (2011)

17. Mackenzie, K.C.H., Xu, P.: Lie Bialgebroids and Poisson Groupoids. Duke Math. J. 73(2), 415-452 (1994)

18. Mokri, T.: Matched Pairs of Lie Algebroids. Glasg. Math. J. 39(2), 167-181 (1997)

19. Pradines, J.: Représentation des jets non holonomes par des morphismes vectoriels doubles soudés. C. R. Acad. Sci. Paris Sér. A 278, 1523-1526 (1974)

20. Pradines, J.: Fibrés vectoriels doubles et calcul des jets non holonomes, vol 29 of Esquisses Mathématiques [Mathematical Sketches]. Université d'Amiens U.E.R. de Mathématiques, Amiens (1977)

21. Pradines, J.: Remarque sur le groupoïde cotangent de Weinstein-Dazord. C. R. Acad. Sci. Paris Sér. I Math. 306(13), 557-560 (1988)

22. Quillen, D.: Superconnections and the Chern Character. Topology 24(1), 89-95 (1985)

23. Roytenberg, D.: Courant Algebroids, Derived Brackets and Even Symplectic Supermanifolds. ProQuest LLC, Ann Arbor, MI, 1999. Thesis (Ph.D.), University of California, Berkeley

24. Yu, A.: Vauntrob. Lie Algebroids and Homological Vector Fields. Uspekhi Mat. Nauk. 52(2(314)), 161-162 (1997)

25. Voronov, Th. Th.: Graded manifolds and Drinfeld doubles for Lie bialgebroids. In: Quantization, Poisson brackets and beyond (Manchester, 2001), vol 315 of Contemp. Math. American Mathematical Society, Providence, RI, pp. 131-168 (2002)

26. Voronov, Th. Th.: Q-Manifolds and Mackenzie Theory. Commun. Math. Phys. 315(2), 279-310 (2012) 